\title{
KINEMATICS AND THERMODYNAMICS ACROSS A PROPAGATING NON-STOICHIOMETRIC OXIDATION \\ PHASE FRONT IN SPENT FUEL GRAINS
}

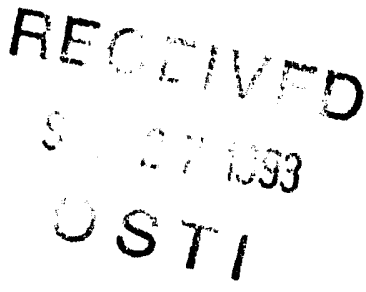

\author{
R.B. Stout \\ E.J. Kansa \\ A.M. Wijesinghe \\ This paper was prepared for submittal to the \\ ASME Micromech. Random Media \\ Charlottesville, VA \\ June 6-9, 1993
}

September 1993

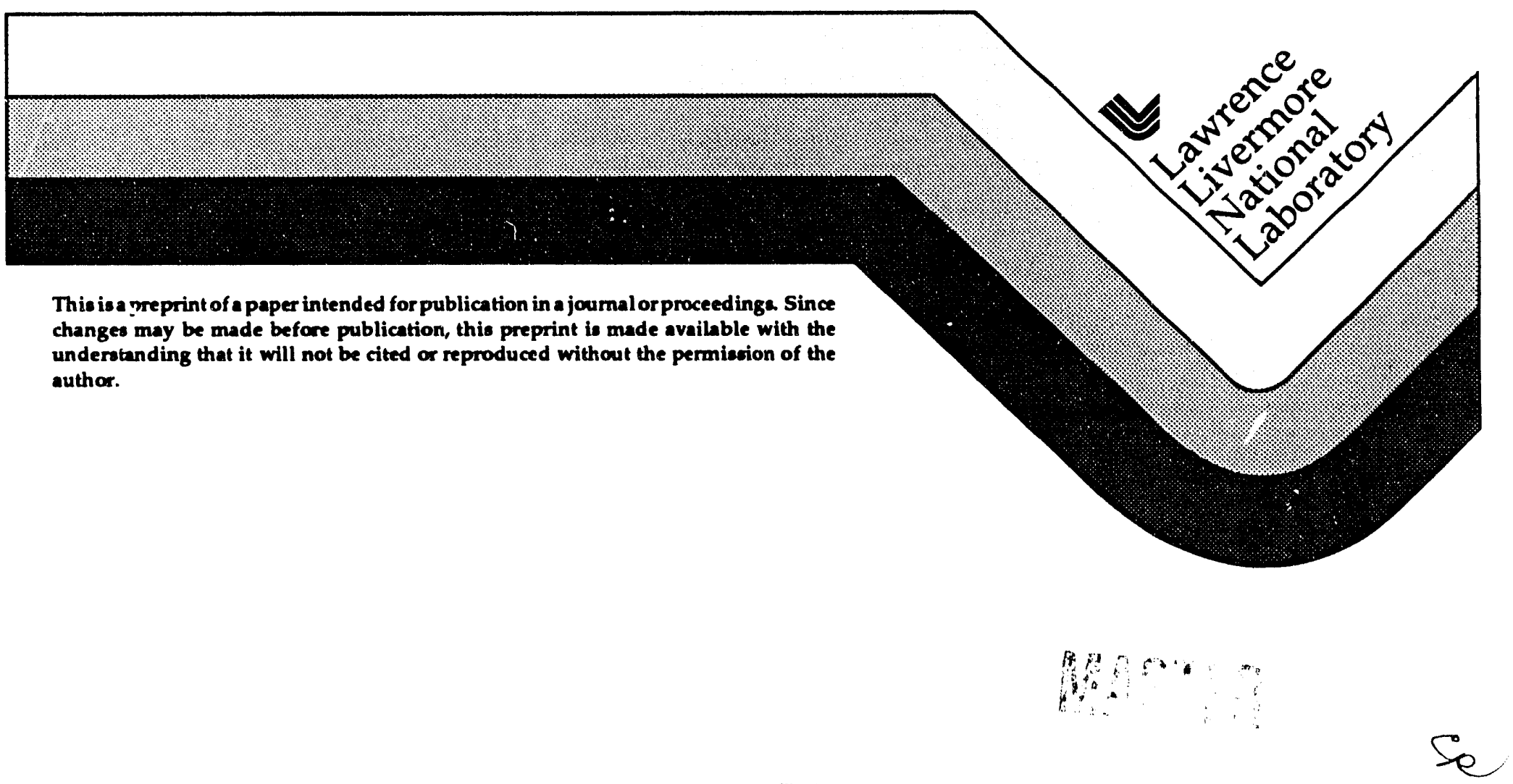





\title{
KINEMATICS AND THERMODYNAMICS ACROSS A PROPAGATING NON-STOICHIOMETRIC OXIDATION PHASE FRONT IN SPENT FUEL GRAINS
}

\author{
R B Stout, E J Kansa, A M Wijesinghe \\ Univ. of California/LLNL, P.O. Box 808, Livermore, CA 94551
}

\begin{abstract}
Spent fuel from power reactors contains mixtures, alloy subsets, and compounds of elements; but the aggregate atomic densities in spent fuel are dominated by uranium and oxygen atoms. With the exception of some $\mathrm{UO}_{2}$ fuels with burnable poisons (primarily gadolinia in BWR rods), the other elements with significant atomic densities in spent fuel evolve during reactor operation from neutron reactions and fission plus fission decay events. Due to nuclear decay processes, the intrinsic chemical composition and activity of spent fuel will continue to evolve after it is removed from reactors as its radioactivity decays over time. During the time interval when the radioactivity levels are significant, which is the time interval relevant for design and for performance assessment of a geological repository, it is important to develop an understanding and to develop models that describe potential chemical responses in spent fuel and its potential degradational impacts on repository design and performance. One such potential impact is the oxidation response of spent fuel. The oxidation of spent fuel results in an initial phase change of the $\mathrm{UO}_{2}$ lattice to a $\mathrm{U}_{4} \mathrm{O}_{9}$ lattice, and the next phase change is probably to $\mathrm{U}_{3} \mathrm{O}_{8}$ although it has not been observed yet at low temperatures $\left(<200^{\circ} \mathrm{C}\right)$. The $\mathrm{U}_{4} \mathrm{O}_{9}$ lattice is non-stoichiometric with a oxygen to uranium weight ratio $(\mathrm{O} / \mathrm{U})$ at -2.4 . Preliminary indications are that the $\mathrm{UO}_{2}$ has a $\mathrm{O} / \mathrm{U}$ of $\sim 2.4$ at the time just before it transforms into the $\mathrm{U}_{4} \mathrm{O}_{9}$ phase. ${ }^{[1,2]}$ Also, in :he oxygen weight gain vers $u S$ time response, a plateau appears as the $O / U$ approaches $\sim 2.4$. Part of this plateau response is cue to geometrical effects of a $\mathrm{U}_{4} \mathrm{O}_{9}$ phase change front propagating into $\mathrm{UO}_{2}$ grain volumes. However, the plateau time response may be indicative of a metastable phase change delay kinetics or a diffusionai related delay time until the oxygen density can attain a critical value to satisfy the stoichiometry and energy conditions for phase changes. In a previous paper and as a first step, a kinematic and thermodynamic ayalysis was developed to model spatially homogeneous oxidation phase transitions. [3] However, the experimental data clearly show a front of $\mathrm{U}_{4} \mathrm{O}_{9}$ lattice structure propagating into grains of the $\mathrm{UO}_{2}$ lattice structure. To describe this spatially inhomogeneous oxidation phase transition, as well as the expected $\mathrm{U}_{3} \mathrm{O}_{8}$ phase transition from the $\mathrm{U}_{4} \mathrm{O}_{9}$ lattice, lattice models are developed and spatially discontinuous kinematic and energetic expressions are derived. The approach will use concepts from statistical mechanics, discontinuum mechanics, and non-equilibrium thermodynamics. In addition, analytical techniques from shock wave analysis will be used to derive the surface discontinuity energetic expressions across the propagating oxidation phase front.
\end{abstract}

\section{INTRODUCTION}

\section{Spent Fuel Oxidation}

In this paper, oxidation phase transition results derived for homogeneous transformations ${ }^{[3]}$ of $\mathrm{UO}_{2}$ to $\mathrm{U}_{4} \mathrm{O}_{9}$ and $\mathrm{U}_{3} \mathrm{O}_{8}$ are extended for the case of inhomogeneous transformations within grain volumes of $\mathrm{UO}_{2}$ spent fuel. The inhomogeneous transformation of $\mathrm{UO}_{2}$ to $\mathrm{U}_{4} \mathrm{O}_{9}$ is observed as a boundary front of $\mathrm{U}_{4} \mathrm{O}_{9}$ propagating into a grain of $\mathrm{UO}_{2}$. In the case of $\mathrm{U}_{4} \mathrm{O}_{9}$ transforming into $\mathrm{U}_{3} \mathrm{O}_{8}$, it is believed that a boundary front of $\mathrm{U}_{3} \mathrm{O}_{8}$ will also propagate into a grain of $\mathrm{U}_{4} \mathrm{O}_{9}$. For some tempe ature histories, it may be possible to have both types of phase fronts propagating simultaneously into a grain, or the $\mathrm{U}_{4} \mathrm{O}_{9}$ phase front be over taken by the $\mathrm{U}_{3} \mathrm{O}_{8}$ phas 4 front and disappear. These latter two cases will not be treated herein; only the isolated cases for a grain with a $\mathrm{UO}_{2}$ and $\mathrm{U}_{4} \mathrm{O}_{9}$ phase front or for a grain with a $\mathrm{U}_{4} \mathrm{O}_{9}$ and $\mathrm{U}_{3} \mathrm{O}_{8}$ will be analyzed.

The notation and equations from the previous analysis of homogeneous phase transformation will be briefly reviewed in the following section. Essentially, the analysis is microscopic and 
formulates the spent fuel oxidation problem as both kinematically and energetically dependent on lattice structure. The lattice structure contains associated lattice atoms and vacancies from fission events plus the addition of oxygen atoms responsible for the higher oxidization phase transitions from $\mathrm{UO}_{2}$ spent fuel. Following this brief review, kinematic and energetic equations across propagating oxidation fronts will be developed. The energetic equation includes terms for the non-stoichiometric effects of oxygen in the lattice structure. In describing the evolution of the microscopic lattice structure through the phase changes, the mathematical description does not use the crystallographic lattice structure, but will use an idealized pseudo-lattice structure that has four uranium atoms per cell. Thus, a $\mathrm{UO}_{2}$ cell is identified as $\mathrm{U}_{4} \mathrm{O}_{8}$, and a cell of $\mathrm{U}_{4} \mathrm{O}_{9}$ forms from a $\mathrm{U}_{4} \mathrm{O}_{8}$ cell by the reaction of one additional oxygen atom. Finally, the $\mathrm{U}_{3} \mathrm{O}_{8}$ would be $\mathrm{U}_{4} \mathrm{O}_{32 / 3}$ (or $\mathrm{U}_{4} \mathrm{O}_{10.67}$ ) and a $\mathrm{U}_{4} \mathrm{O}_{9}$ cell would become $\mathrm{U}_{4} \mathrm{O}_{10.67}$ by the reaction of 1.67 (on the average) additional oxygen atoms. In the idealized development, there is a distinction between the oxygen in a lattice structure and the amount of additional oxygen in the lattice. For example, it is experimentally observed for spent fuel that the $\mathrm{U}_{4} \mathrm{O}_{9}$ crystallographic lattice structure has an additional $\sim 0.6$ oxygen atoms (i.e., it has a nonstoichiometric oxygen to uranium atomic (O/U) ratio of 2.4 ). This excess atomic oxygen concentration has both kinematic and energetic dependences in the thermodynamic equations. Model development and additional experiments are necessary to clarify these dependences, and to address their impact on spent fuel performance in a repository environment.

\section{REVIEW OF HOMOGENEOUS SPENT FUEL OXIDATION MODEL}

\section{Kinematics of Deformation}

A model describing the deformation response of a contiguous set of lattice cells was developed. [3] The model represented a lattice cell by a set of vector attributes. These vector attributes became the variables in density functions for the number of lattice cells of a particular species per unit volume. The lattice density was a stochastic function and species-dependent integrals over the set of vector attributes provide probable values for the deformation response. The deformation response was derived based on an idealized unit cell lattice structure that has an effective height, width, and depth. At any time, attribute vectors denoted by $(\underline{a}, \underline{b}, \underline{c})$ can be assigned to describe the cell's dimensions, and in a reference configuration of deformation these vector attributes can be selected as a spatially local right-handed set of coordinates. A set of $(\underline{a}, \underline{b}, \underline{c})$ vector attributes can be assigned to every lattice cell in a spatial domain of volume $R_{0}$ enclosed by a boundary $\partial R_{0}$. To represent a stochastic motion of deformation at time $t$, a set of velocity vector attributes $(\underline{\dot{a}}, \underline{\dot{b}}, \underline{\dot{c}})$ was also assigned to each lattice cell. Then, the probable number of cells at time $t$ per unit spatial volume neighborhood of point $x$ per unit vector attribute volume neighborhood of species point $(\underline{a}, \underline{b}, \underline{c}, \underline{\dot{a}}, \underline{\dot{b}}, \underline{\dot{c}})$ is denoted by a density function $\mathrm{P}_{\mathrm{MN}}(x, t$, $\underline{a}, \underline{b}, \underline{c}, \underline{\dot{a}}, \underline{\dot{b}}, \underline{\dot{c}})$ where $\mathrm{MN}$ denotes a specific lattice phase. For shorthand purposes, the species space for the six vector attributes $\{\underline{a}, \underline{b}, \underline{c}, \underline{\dot{a}}, \underline{\dot{b}}, \underline{\dot{c}}\}$ was denoted as $\{q\}$, or domain $\mathrm{Q}$; i.e., lattice cell species $q$ is an element in the lattice cell species domain $Q$. In order to identify and describe the evolution of different lattice structures for the three likely phases of $\mathrm{UO}_{2}$, three different density functions are defined: $\mathrm{P}_{48}(\underline{x}, t, q)$ denotes the number density of $\mathrm{U}_{4} \mathrm{O}_{8}$ (i.e., $\mathrm{UO}_{2}$ ) lattice cells such that each cell contains four uranium atoms, $\mathrm{P}_{49}(x, t, q)$ denotes the number density of $\mathrm{U}_{4} \mathrm{O}_{9}$ lattice cells such that each cell contains four uranium atoms, and $\mathrm{P}_{38}(x, t, q)$ denotes the number density of $\mathrm{U}_{3} \mathrm{O}_{8}$ (this phase will be denoted as $\mathrm{U}_{3} \mathrm{O}_{8}$ rather that $\mathrm{U}_{4} \mathrm{O}_{32 / 3}$ ) lattice cells such that each cell contains four uranium atoms, for point $x$ in $\mathrm{R}_{\mathrm{o}}+\partial \mathrm{R}_{\mathrm{o}}$ at time $t$.

The density functions $\mathrm{P}_{48}, \mathrm{P}_{49}$, and $\mathrm{P}_{38}$ can each be decomposed into two functions: one is for a "continuum-like" deformation response and the other is for a "discontinuum-like" deformation response. The decomposition functions are defined as $\left.\left.P_{48} \equiv P_{48} \mid+P_{48}\right], \quad P_{49} \equiv P_{49} \mid+P_{49}\right]$, $\left.P_{38} \equiv P_{38} \mid+P_{38}\right]$, where the single bar symbol denotes the continuum response and the single square bracket denotes the discontinuum response. This decomposition is used to analytically represent the annihilation and creation rate changes in cell density when phase transformations occur. With respect to the deformation response description, the phase transformation from existing lattice cells to new cell sizes is represented as a discontinuum deformation because a lattice species $q$ deforms 
discontinuously into a species $q^{*}$ that has finitely different dimensions within an arbitrarily small increment of time. Thus, a discontinuum lattice response contrasts to a continuum lattice response which has lattice species $q$ deforming into a species $q^{*}$ that has infinitesimally different dimensions within an arbitrarily small increment of time. In addition to lattice cell deformations, spent fuel contains fission product elements (including neutron reaction products (actinides)) that reside in interstitial and substitutional atomic lattice positions, vacancies (missing atoms), and for oxidation processes to occur, oxygen atoms; these four kinds of densities are aggregated and denoted by four density functions, $\left.\left.\left.\mathrm{P}_{I}\right], \mathrm{P}_{S}\right], \mathrm{P}_{V}\right]$, and $\left.\mathrm{P}_{\mathrm{O}}\right]$, respectively. The function arguments of these four density functions are similar to the lattice density functions, except the deformation is incrementally discontinuous by incremental vector attributes ( $\underline{\hat{a}}, \underline{\hat{b}}, \underline{\hat{c}}$, ) as interstitial, substitutional, vacancy, and oxygen species migrate in and out of the lattice. Thus, their arguments are $(\underline{x}, t, \underline{a}, \underline{b}, \underline{c}$, $\left.\underline{\hat{a}}, \underline{\hat{b}}, \hat{\underline{c}}, \underline{v}_{K}\right)$ where $\underline{x}$ is position, $t$ is time, $(a, \underline{b}, \underline{c})$ is the local lattice cell size, ( $\underline{\hat{a}}, \underline{\hat{b}}, \underline{\hat{c}}$,) is the local incremental change in lattice vectors $(\underline{a}, \underline{b}, \underline{c})$ as a corresponding interstitial atom, substitutional atom, vacancy, or oxygen atom occupies a local position in a lattice cell, and $\underline{v}_{K}$ is the species velocity relative to the local lattice cell velocity $(K \sim I, S, V$, or $O$ species). For shorthand, the previous set $\mathrm{q}$ vector attributes and space $Q$ will be augmented with the attribute set ( $\underline{\hat{a}}, \underline{\hat{b}}, \underline{\hat{c}}, \underline{Y}_{K}$ ) and all density functions will have the same arguments set; $(x, t, q)$.

Using the above definitions and lattice structure descriptors, a function for the deformation response of a material body can be developed in terms of lattice scale attributes. For this development consider two spatial points, say $\underline{x}_{A}$ and $\underline{x}_{B}$ in a material $\mathrm{R}_{\mathrm{o}}$. To start, assume that the lattice cell structure is perfect and has only $\mathrm{UO}_{2}$ lattices. Then, the probable total relative velocity $\underline{v}\}_{A}^{B}$ of point $B$ relative to point $A$ at time $t$ is

$$
\begin{aligned}
&\left.v_{i}\right\}_{A}^{B}=\int_{\underline{x}_{A} Q}^{x_{Q}} \int_{a_{i}} e_{j k m} b_{k} c_{m}+\dot{b}_{i} e_{j k m} c_{k} a_{m} \\
&\left.+\dot{c}_{i} e_{j k m} a_{k} b_{m}\right) P_{48}(\underline{\xi}, t, q) \\
&+\left(a_{i} e_{j k m} b_{k} c_{m}+b_{i} e_{j k m} c_{k} a_{m}+c_{i} e_{j k m} a_{k} b_{m}\right)
\end{aligned}
$$

$$
\times \dot{\bar{P}}_{48}(\underline{\xi}, t, q) d q d \xi_{j}
$$

which is the contribution to velocity from all lattice species (integration over $Q$ ) of volume elements (example, $d \xi_{j} e_{j k m} b_{k} c_{m}$ ) along an arbitrary path from spatial point $\underline{x}_{A}$ to $\underline{x}_{B}$, where the total time rate of change for $\dot{\bar{P}}_{48}$ is defined as

$$
\dot{\bar{P}}_{48} \equiv \partial_{i} P_{48}+\partial_{i}\left(v_{i} P_{48}\right)
$$

For notational shorthand purposes, equation (2-1a) can be written in terms of functional operators as

$$
\left.\left.\left.v_{i}\right\}_{A}^{B} \equiv \dot{L}_{i j 48} P_{48} \Delta x_{j}\right\}_{A}^{B}+L_{i j 48} \dot{\bar{P}}_{48} \Delta x_{j}\right\}_{A}^{B}
$$

where the definitions for operators $\left.\dot{L}_{i j 48}() \Delta x_{j}\right\}_{A}^{B}$ and $\left.L_{i j 48}() \Delta x_{j}\right\}_{A}^{B}$ follow from equation (2-1a) and the $\left.\Delta x_{j}\right|_{A} ^{B}$ is a shorthand for indicating spatial integration from point $\underline{x}_{A}$ to point $\underline{x}_{B}$.

To include relative lattice velocities from oxidation phase changes, the lattice density rates for functions $P_{\mathrm{NM}}$ are decomposed into continuum and discontinuum terms. In addition, the relative velocity of equation (2-2) for lattice motion is also dependent on oxygen, interstitial, substitutional, and vacancy density changes in the lattice cells along the line integral path between atoms $A$ and $B$. As remarked previously, the incremental finite deformation is $(\underline{\hat{a}}, \underline{\hat{b}}, \underline{\hat{c}})$, which is represented in the relative velocity with operator

$$
\begin{aligned}
L_{i j K}(\cdot) & \equiv \int_{Q}\left(\hat{a}_{i} e_{j k m} b_{k} c_{m}+\hat{b}_{i} e_{j k m} c_{k} a_{m}\right. \\
& \left.+\hat{c}_{i} e_{j k m} a_{k} b_{m}\right)_{K}(\cdot) d q
\end{aligned}
$$

where subscript $\mathrm{K}$ identifies the operator function as oxygen (O), interstitial (I), substitutional (S) and vacancy (V). Then, the relative velocity of equation (2-2) in an oxidizing $\mathrm{UO}_{2}$ spent fuel lattice can be decomposed into continuum and discontinuum components that includes the added dependence on oxygen, interstitial, substitutional, and vacancy densities:

$$
\begin{gathered}
\left.\left.v_{i}\right\}_{A}^{B}=\left.v_{i}\right|_{A} ^{B}+v_{i}\right]_{A}^{B} \\
\left.\left.v_{i}\right|_{A} ^{B}=\left(\dot{L}_{i j 48} P_{48}\left|+L_{i j 48} \dot{\bar{P}}_{48}\right|\right) \Delta x_{j}\right\}_{A}^{B}
\end{gathered}
$$




$$
\left.\left.\left.\left.v_{i}\right]_{A}^{B}=\left(L_{i j 48} \dot{\bar{P}}_{48}\right]+L_{i j K} \dot{\bar{P}}_{K}\right]\right) \Delta x_{j}\right\}_{A}^{B}
$$

where continuum and discontinuum relative velocities $v_{i} \mid$ and $v_{i}$ ] are defined in terms of the lattice cell decomposition functions $P_{48} \mid$ and $\left.P_{48}\right]$ plus the $\mathrm{K}$ letter index for the four letters $\mathrm{O}, \mathrm{I}, \mathrm{S}$, and $\mathrm{V}$ to obtain relative velocity contributions from oxygen, interstitial, substitutional, and vacancy density changes. The latter density functions have a time rate given by

$$
\left.\left.\left.\dot{\bar{P}}_{K}\right] \equiv \partial_{i} P_{K}\right]+\partial_{i}\left(\left(v_{i}+v_{K i}\right) P_{K}\right]\right)
$$

The total velocity of position $B$ is the velocity of position A plus the relative velocity, i.e.,

$$
\left.v_{i}(B, t)=v_{i}(A, t)+v_{i}\right\}_{A}^{B}
$$

where $v_{i}(A, t)$ is the velocity of position A with respect to an arbitrary frame of reference.

From equation (2-4), a relative deformation response can be written for the position of point $B$ relative to point $A$ by performing an integration over time. This integration requires an initial condition at $\mathrm{t}=0$ (which is introduced by selecting a reference coordinate frame) and a time duration, say $\mathrm{t}=0$ to to $\mathrm{t}=\Delta \tau$ where $\Delta \tau$ is an arbitrary future time. At time $\Delta \tau$ and for contiguous lattice cell species densities $P_{M N}$ and point species $P_{K}$ ], the total relative deformation of point $B$ relative to point $\mathrm{A}$, denoted by $\underline{\chi}\}_{A}^{B}$, is given by

$$
\begin{aligned}
\left.\chi_{i}\right\}_{A}^{B} & \left.\left.=\mathrm{X}_{i}\right\}_{A}^{B}+\int_{O}^{\Delta \tau} v_{i}\right\}_{A}^{B} d \tau \\
\left.=\mathrm{X}_{i}\right\}_{A}^{B} & +\left(\dot{L}_{i j M N} P_{M N}\left|+L_{i j M N} \dot{\bar{P}}_{M N}\right|+L_{i j M N} \dot{\bar{P}}_{M N}\right] \\
& \left.\left.\left.+L_{i j K} \dot{\bar{P}}_{K}\right]\right) \Delta x_{j}\right\}_{A}^{B} \Delta \tau
\end{aligned}
$$

where the $\left.L_{i j M N}() \Delta x_{j}\right\} \Delta \tau$ and $\left.L_{i j K}() \Delta x_{j}\right\} \Delta \tau$ functional operators include a $\Delta \tau$ to denote integration over time, as indicated in equation (25a), and $\underline{X}\}_{A}^{B}$ is the initial position vector of point $B$ relative to point $A$ at time $t=0$. In equation (2$5 b)$, the total relative deformation is decomposed into continuum and discontinuum functionals, which are defined over independent function spaces containing $\left.\mathrm{P}_{\mathrm{MN}} \mid, \mathrm{P}_{\mathrm{MN}}\right]$ and $\left.\mathrm{P}_{\mathrm{K}}\right]$. For the thermodynamic model of the next section, a strain tensor derived from only the continuum part of the deformation is used as a thermodynamic function argument, and it can be shown to be given by (see [4-5] for analog derivations)

$$
\begin{aligned}
2 \gamma_{i j} \mid & =\left(\dot{L}_{i j M N}+\dot{L}_{j M N}\right) P_{M N} \mid \Delta \tau+\left(L_{i j M N}+L_{j i M N}\right) \\
& \times \dot{\bar{P}}_{M N} \mid \Delta \tau+\text { second order terms } \\
=( & (2-6 \mathrm{a}) \\
& \left.-P_{i j M N}+L_{j M N}\right)\left(P_{M N}(\underline{x}, o, t, q) \mid\right.
\end{aligned}
$$

where equation (2-6b) is a time integration of the total time derivative of $\left.\left(L_{i j M N} P_{M N}\right)\right)$ over the time interval $(o, t)$ to give a time integrable strain measure relative to an idealized "unstrained" reference lattice cell set $\left\{q_{o}\right\}$ that is continuum-like for each phase MN. For the present analysis, only the linear terms of $P_{M N} \mid$ and $\dot{\bar{P}}{ }_{M N} \mid$ will be retained, then the gradient of the continuum relative velocity of equation (2-4b) will be equal to the time derivative of the linear strain tensor in equation (26 ). This completes a review of kinematic measures for lattice deformations that will be used in the following section on thermodynamic modelling.

\section{Energetics of Homogeneous Phase Transformation Thermodynamics}

A nonequilibrium thermodynamic model will be reviewed for the rate of entropy production during homogeneous oxidation phase transformations of spent fuel.[3] The model represented the internal energy change terms that arise from the stressstrain work for a deforming lattice structure, mass transport, and from phase transformation energies between homogeneous lattice structures. From the entropy rate response and an Onsager thermodynamic formulation, [6] an oxidation response functional was written that expressed the kinetics of phase changes as functionally dependent on the thermodynamic force for transport and the thermodynamic force for phase change energetics. This representation was for a spatially continuous phase change process. Additional analysis will be completed in a later section to extend the following thermodynamic expressions to address a phase change front that propagates as a spatial surface of discontinuity between two phases. 
To begin this review, assume that an internal energy functional, $\bar{\varepsilon}$, exists (energy per unit spatial volume) that has function arguments of entropy $\eta(x, t)$, continuum strain $\underline{\gamma}(\underline{x}, t) \mid$, the three lattice phase density functions $\left.\left.P_{48}(x, t, q)\right], P_{49}(x, t, q)\right]$, $\left.P_{38}(x, t, q)\right]$, and the four point density functions for atom and vacancy density concentrations, $\left.\left.\left.\left.P_{\mathrm{O}}(x, t, q)\right], P_{\mathrm{I}}(x, t, q)\right], P_{\mathrm{S}}(x, t, q)\right], P_{\mathrm{V}}(x, t, q)\right]$;

$$
\begin{aligned}
& \left.\left.\left.\left.\left.P_{\mathrm{O}}\right], P_{\mathrm{I}}\right], P_{\mathrm{S}}\right], P_{\mathrm{V}}\right]\right) \\
& \equiv \varepsilon(f)
\end{aligned}
$$$$
\left.\left.\bar{\varepsilon}(x, t)=\varepsilon\left(\eta, \underline{\underline{\gamma}} \mid, P_{48}\right], P_{49}\right], P_{38}\right],
$$

where $f$ is shorthand to replace the writing of the nine functions. The spatial domain of points $x$ is $R_{\mathrm{o}}+\partial R_{\mathrm{o}}$, the species domain of $q$ is $\{q\}$ or $Q$, and the time domain is time $t$ greater than an initial time at $t=0$. In addition to an existence of $\varepsilon$, a functional form will be assumed for the internal energy in terms of functional operators that effectively integrate out the explicit dependence of the variables $q$ in $\varepsilon$ to obtain $\bar{\varepsilon}(x, t)$. This energy functional form is an analytical equivalent of the physical problem of having a space domain $R_{\mathrm{o}}+$ $\partial R_{0}$ at time $t$ filled with various possible compositions of lattice densities of type $P_{48}, P_{49}$, and $P_{38}$, with their associated energies, and into which oxygen atoms, interstitial atoms, substitutional atoms, and vacancies defect densities of $P_{\mathrm{O}}, P_{\mathrm{I}}, P_{\mathrm{S}}, P_{\mathrm{V}}$, respectively, are inserted with their associated energies. This can be written as

$$
\begin{aligned}
& \bar{\varepsilon}(x, t)\left.=\varepsilon(f) \equiv \Delta P_{48} \varepsilon(f) P_{48}\right] \\
&\left.\left.+\Delta P_{49} \varepsilon(f) P_{49}\right]+\Delta P_{38} \varepsilon(f) P_{38}\right] \\
&\left.\left.+\Delta P_{\mathrm{O}} \varepsilon(f) P_{\mathrm{O}}\right]+\Delta \mathrm{P}_{\mathrm{I}} \varepsilon(f) P_{\mathrm{I}}\right] \\
&\left.\left.+\Delta P_{\mathrm{S}} \varepsilon(f) P_{\mathrm{S}}\right]+\Delta P_{\mathrm{V}} \varepsilon(f) P_{\mathrm{V}}\right]
\end{aligned}
$$

where the $\Delta_{()} \varepsilon$ denote thermodynamic chemical potential energies that are functional operators (integrates over domain $Q$ ) defined from the internal energy functional. Note that the above is an analytical statement that the internal energy at a point and time is the energy of the lattice species plus the energy of the atoms/vacancies added to that lattice. Also, note that the above energy expression does not explicitly represent any oxide and other compounds plus metal alloy clusters that may form within the aggregate set of fission product elements However, if such oxides/ compounds in spent fuel exist before and after a uranium oxidation phase transformation, then their energy change effect on the kinetics of the uranium phase transformation would not be an explicit net energy term in an energy change rate expression. Thus, while equation (3-2) represents a physical view of lattice plus atom/vacancy energy contributions to the internal energy functional, it also illustrates a significant lack of detailed knowledge of the fission product sub-energies that exist within the possible uranium oxide phases of spent fuel. This lack of detailed knowledge can only be augmented and completed by experimental methods; however, for spent fuel oxidation response within the expected environmental timehistories of geological repositories, the above internal energy assumption represents an initial starting point in model development that also may be sufficient. Using repeated subscripts, equation (3-2) is written

$$
\left.\left.\varepsilon(f)=\Delta_{P_{\mathrm{NM}}} \varepsilon P_{\mathrm{NM}}\right]+\Delta_{P_{\mathrm{K}}} \varepsilon P_{\mathrm{K}}\right]
$$

where the repeated subscript (NM) denotes a sum over the four lattice density functions and the repeated subscript $(K)$ denotes a sum over the three atomic and the vacancy functions.

For quasi-static deformation processes (no kinetic energy/acceleration terms) in spatial domains that do not have a propagating surface of discontinuity, the first law of thermodynamics sets the rate of change of internal energy equal to the rate of work (body force plus stress), $\dot{\bar{W}}$, plus the rate of heat change, $\dot{\bar{H}}$, for any spatial subset $R+$ $\partial R$ in $R_{\mathrm{o}}+\partial R_{\mathrm{o}}$

$$
\dot{\bar{\varepsilon}}=\dot{\bar{W}}+\dot{\bar{H}}
$$

The rate of heat exchange for spatial domain $R+$ $\partial R$ is

$$
\dot{\bar{H}}=\int_{R} \dot{H}_{o} d \underline{x}+\int_{\partial R} h_{i} n_{i} d \underline{x}
$$

where $H_{o}$ is the heat generation rate at points in $R$ and $h_{i}$ is the heat flux vector at points on boundary $\partial R$, and $n_{i}$ is the outward directional unit normal vector at points on boundary $\partial R$. The rate of work on spatial domain $R+\partial R$ is 


$$
\dot{\bar{W}}=\int_{R} f_{j} v_{j} d \underline{x}+\int_{\partial R} \sigma_{i j} n_{i} v_{j} d \underline{x}
$$

where $f_{i}$ is the body force vector, $v_{i}$ is the velocity vector, and $\sigma_{i j}$ is the stress tensor. The rate of internal energy change for the spatial domain $R+$ $\partial R$ is

$$
\begin{aligned}
\dot{\bar{\varepsilon}}= & \int_{R} \partial_{i} \varepsilon(f) d \underline{x}+\int_{\partial R} \varepsilon(f) v_{i} n_{i} \\
& \left.+\Delta_{P_{K}} \varepsilon(f) P_{K}\right] v_{K_{i}} n_{i} d \underline{x}
\end{aligned}
$$

where the first integral is the time rate of change of internal energy at spatial points in $R$ and the second integral is the flux of internal energy through boundary $\partial R$ at the local velocity of the lattice $\left(v_{i} n_{i}\right)$ plus the flux of internal energy from the oxygen, interstitial, substitutional, and vacancies density functions at their relative velocity $\left(v_{K} \cdot \underline{n}\right)$ with respect to the lattice velocity $v_{i}$. The surface integral expression in equations (3-5), (3-6), and (3-7) over $\partial R$ can be rewritten as volume integrals by applying the divergence theorem of integral calculus, providing that the integrands are spatially continuous functions within domain $R$. This is the assumed case for the present analysis. Therefore, equations (3-5), (3-6), and (3-7) can be substituted into equation (3-4) from which, along with the velocity expression of section 2 and the use of the divergence theorem, one obtains the following form of equation (3-4) as a volume integral over $R$ :

$$
\begin{aligned}
& \int_{R}\left(\Delta_{\eta} \varepsilon \dot{\eta}-\dot{H}_{o}-\partial_{i} h_{i}\right) d \underline{x} \\
& =\int_{R}\left(\left(f_{j}+\partial_{i} \sigma_{i j}\right) \cdot v_{j}+\sigma_{i j} \partial_{i} v_{j}-\Delta_{\gamma_{i}} \varepsilon \dot{\gamma}_{i j} \mid\right. \\
& \left.\left.\quad-\Delta P_{N M} \varepsilon \dot{\bar{P}}_{N M}\right]-\Delta P_{K} \varepsilon \dot{\bar{P}}_{K}\right] \\
& \left.\left.\quad-\partial_{i}\left(\Delta P_{K} \varepsilon\right) P_{K}\right] v_{K i}\right) d x
\end{aligned}
$$

The term on the left side of equation (3-8) is the total entropy rate energy minus heat; this net energy rate is the rate of entropy dissipation/ production energy; and will be denoted here by

$\Delta_{\eta} \varepsilon \dot{\eta}^{*}$. For nonequilibrium thermodynamic processes, the entropy dissipation rate is nonnegative. The first term on the right side of equation (3-8) contains the stress equilibrium expression, which must be zero because the velocity is arbitrary, hence

$$
f_{j}+\partial_{i} \sigma_{i j}=0 \text { for all } \underline{x} \text { in } R
$$

The remaining terms in equation (3-8) are internal energy rates associated with the stress-velocity gradient work rate, strain energy rate, phase transformation rates, and mass transport rates. Using the above entropy dissipation/production function, $\eta^{*}$, equations (2- 4b), (2-6), and (3-9), and the assumptions of small continuum strains

(i.e., neglect higher order strain terms in $\dot{\gamma}_{i j} l$ ), equation (3-8) can be written as

$$
\begin{gathered}
\int_{R} \Delta_{\eta} \varepsilon \dot{\eta}^{*} d \underline{x}=\int_{R}\left(\left(\sigma_{i j}-\Delta_{\gamma i j} \varepsilon\right) \dot{\gamma}_{i j} 1\right) \\
\left.\left.+\sigma_{i j} L_{i j_{N}} \dot{\bar{P}}_{\mathrm{NM}}\right]+\sigma_{i j} L_{i j j_{K}} \dot{\bar{P}}_{\mathrm{K}}\right] \\
\left.\left.-\Delta_{P_{N M}} \varepsilon \dot{\bar{P}}_{\mathrm{NM}}\right]-\Delta_{P_{K}} \varepsilon \dot{\bar{P}}_{\mathrm{K}}\right] \\
\left.\left.-\partial_{i}\left(\Delta_{P_{K}} \varepsilon\right) P_{\mathrm{K}}\right] v_{K i}\right) d \underline{x}
\end{gathered}
$$

In equation (3-10), the rate of entropy production energy has four generic possible energy rate contributions, the sum of which must not be nonnegative. The energy rate contributions have thermodynamic processes identified with strain rate

processes $\dot{\gamma}_{i j}$ l, lattice-phase change rate processes $\left.\dot{\bar{P}}_{\mathrm{NM}}\right]$, atom/vacancy density rate change process $\left.\dot{\bar{P}}_{\mathrm{K}}\right]$, and atom/vacancy density flux processes $\left.P_{\mathrm{K}}\right] v_{\mathrm{K}_{i}}$. In this analysis, as well as the following surface of discontinuity analysis, all continuum strain rate processes are assumed to occur at thermodynamic equilibrium, hence

$$
\sigma_{i j}=\Delta_{\gamma i j \mid} \varepsilon
$$

and this eliminates the strain rate term in equation (3-10). More importantly, this assumption also means, for modeling purposes here, that all homogeneous phase change kinetic processes are kinematically accessible; i.e., the deformed configuration of a possible phase transformation is not constrained by any prescribed displacement/traction boundary conditions. This assumption does not require that phase transformations occur at zero stress. In fact, the stress state for the $\mathrm{U}_{4} \mathrm{O}_{8}$ to $\mathrm{U}_{4} \mathrm{O}_{9}$ transformation is 
sufficient to form microcracks, and for the $\mathrm{U}_{4} \mathrm{O}_{9}$ to $\mathrm{U}_{3} \mathrm{O}_{8}$ transformation, particles will increase in volume by approximately $30 \%$, which could result in stresses sufficient to form a flaked powder material.

Returning to equation (3-10), consider a possible sequence of oxidation phase of $\mathrm{U}_{4} \mathrm{O}_{8}$ to $\mathrm{U}_{4} \mathrm{O}_{9}$ to $\mathrm{U}_{3} \mathrm{O}_{8}$ for a volume of spent fuel in an arbitrary spatial domain $R$. To transform from $\mathrm{U}_{4} \mathrm{O}_{8}$ to only $\mathrm{U}_{4} \mathrm{O}_{9}$, the lattice rates $\dot{\bar{P}}_{48}$ and $\dot{\bar{P}}_{49}$ are the only non-zero lattice rates. In addition, the interstitial oxygen atomic density in the idealized lattice of $\mathrm{U}_{4} \mathrm{O}_{8}$ has a discontinuous transition in density as the $\mathrm{U}_{4} \mathrm{O}_{9}$ lattice phase is formed during the transformation from $\mathrm{U}_{4} \mathrm{O}_{8}$ to $\mathrm{U}_{4} \mathrm{O}_{9}$. As per the discussion of volumes in a previous section, each volume of a created $\mathrm{U}_{4} \mathrm{O}_{9}$ lattice, with its four uranium atoms of the $\mathrm{P}_{49}$ ] lattice, will remove a $\mathrm{U}_{4} \mathrm{O}_{8}$ lattice volume containing four uranium atoms and one oxygen atom of the $\mathrm{PO}$ ] oxygen density form a $\mathrm{U}_{4} \mathrm{O}_{9}$ lattice; i.e.,

$$
\mathrm{U}_{4} \mathrm{O}_{8}+\mathrm{O} \rightarrow \mathrm{U}_{4} \mathrm{O}_{9}
$$

The mass balance for this phase transformation can also be expressed in terms of rates of change in the lattice density functions $\left.\mathrm{P}_{48}\right]$ and $\mathrm{P}_{49}$ ], and the oxygen density function $P_{0}$ ] relative to the two different lattices as

$$
\begin{aligned}
& \left.\dot{\bar{P}}_{49}\right] \Leftrightarrow-\dot{\bar{F}}_{48} ; \\
& \left.\dot{\bar{P}}_{49}\right] \Leftrightarrow-\dot{\bar{P}}_{\left.\mathrm{O}]_{48}-\dot{\bar{P}}_{\mathrm{O}}\right]_{49}}
\end{aligned}
$$

where (3-13a) functionally expresses a removal rate of $\mathrm{U}_{4} \mathrm{O}_{8}$ lattice (four $\mathrm{UO}_{2}$ lattices) at the rate one $\mathrm{U}_{4} \mathrm{O}_{9}$ lattice is formed and (3-13b) functionally expresses the $\left.\dot{\bar{P}}_{\mathrm{O}}\right]_{48}$ removal rate of all oxygen from the $\mathrm{U}_{4} \mathrm{O}_{8}$ lattice, such that a $\left.\dot{\bar{P}}_{0}\right]_{49}$ rate becomes interstitial oxygen in the $\mathrm{U}_{4} \mathrm{O}_{9}$ lattice and the rest is consumed at a rate equal to the $\dot{\bar{P}}_{49}$ ] rate at which the $\mathrm{U}_{4} \mathrm{O}_{9}$ lattice is formed. In addition to the above two functional conservation expressions, analogous ones are assumed to exist for the interstitial, substitutional, and vacancy density functions as the $\mathrm{U}_{4} \mathrm{O}_{8}$ lattice transforms to a $\mathrm{U}_{4} \mathrm{O}_{9}$ lattice, i.e.,

$$
\left.\left.\left.\dot{\bar{P}}_{\mathrm{I}}\right]_{49} \Leftrightarrow-\dot{\bar{P}}_{I}\right]_{48} ; \dot{\bar{P}}_{\mathrm{S}}\right]_{49} \Leftrightarrow-\dot{\bar{P}}_{S]_{48}} ;
$$

$$
\left.\left.\dot{\bar{P}}_{\mathrm{v}}\right]_{49} \Leftrightarrow-\dot{\bar{P}}_{\mathrm{v}}\right]_{48}
$$

Substituting equation (3-11) and expressions (3-13) into equation (3-10), and considering only the $\mathrm{U}_{4} \mathrm{O}_{8}$ to $\mathrm{U}_{4} \mathrm{O}_{9}$ transformation, the dissipation entropy energy rate can be written as

$$
\begin{aligned}
\int_{R} \Delta_{\eta} \varepsilon \dot{\eta}^{*} & \int_{48}^{49} d \underline{x}=\int_{R}\left(\left(\sigma_{i j} L_{i j}\right]_{48}^{49}\right. \\
& \left.\left.\left.-\sigma_{i j} L_{i j o}\right]_{48}^{49}+\Delta_{P_{48}} \varepsilon+\Delta_{P_{0}} \varepsilon-\Delta_{P_{49}} \varepsilon\right) \dot{\bar{P}}_{49}\right] \\
& \left.\left.\left.+\left(\sigma_{i j} L_{i j o}\right]_{48}^{49}-\Delta_{P_{0}} \varepsilon\right]_{48}^{49}\right) \dot{\bar{P}}_{\mathrm{O}}\right]_{49} \\
& \left.\left.\left.+\left(\sigma_{i j} L_{i j l}\right]_{48}^{49}-\Delta_{P_{i}} \varepsilon\right]_{48}^{49}\right) \dot{\bar{P}}_{\mathrm{I}}\right]_{49} \\
& \left.\left.\left.+\left(\sigma_{i j} L_{i j s}\right]_{48}^{49}-\Delta_{P_{s}} \varepsilon\right]_{48}^{49}\right) \dot{\bar{P}}_{\mathrm{S}}\right]_{49} \\
& \left.\left.\left.+\left(\sigma_{i j} L_{i j v}\right]_{48}^{49}-\Delta_{P_{V}} \varepsilon\right]_{48}^{49}\right) \dot{\bar{P}}_{\mathrm{V}}\right]_{49} \\
& \left.\left.-\partial_{i}\left(\Delta_{P_{K}} \varepsilon\right) P_{K}\right] v_{K i}\right) d \underline{x}
\end{aligned}
$$

where terms such as $\left.\sigma_{i j} L_{i j}\right]_{48}^{49}$ operating on $\dot{\bar{P}}_{49}$ is shorthand notation for the stress work during the $\mathrm{U}_{4} \mathrm{O}_{8}$ to $\mathrm{U}_{4} \mathrm{O}_{9}$ transformation, $\left(\sigma_{i j} L_{i j 49}-\sigma_{i j} L_{i j 48}\right)$ $\times \dot{\bar{P}}_{49}$ ] (the second term results from expression [3-13a]), and terms such as $\left.\Delta_{P_{s}} \varepsilon\right]_{48}^{49}$ operating on $\dot{\bar{P}}_{\mathrm{S}} \mathrm{J}_{49}$ denote the internal energy changes for substitutional atoms in the $\mathrm{U}_{4} \mathrm{O}_{9}$ lattice relative to the $\mathrm{U}_{4} \mathrm{O}_{8}$ lattice, $\left.\left.\left(\Delta_{P_{S}} \varepsilon_{49} \dot{\bar{P}}_{\mathrm{S}}\right]_{49}-\Delta_{P_{S}} \varepsilon_{48} \dot{\bar{P}}_{\mathrm{S}}\right]_{49}\right)$ (the second term results from expression (3-13c)). From equation (3-14), the dissipation entropy rate for $\mathrm{U}_{4} \mathrm{O}_{8}$ transforming to $\mathrm{U}_{4} \mathrm{O}_{9}$ depends on energy terms from the rate of five density functions and four flux density functions. Note that if equation (3-14) were applied to an ideally perfect sample of $\mathrm{U}_{4} \mathrm{O}_{8}$, i.e., no fission products $\left(\mathrm{P}_{S}=\mathrm{P}_{\mathrm{I}}=0\right)$ and stoichiometric phase transformations, then only the rate $\dot{\bar{P}}_{49}$ ] and the flux $\left.\mathrm{Po}_{\mathrm{O}}\right] v_{\mathrm{O}}$ i term would be present as possible terms that contribute entropy energy. Thus, equation (3-14) addresses the 
additional energies, in an aggregate and phenomenological modelling analysis, that occur as $\mathrm{U}_{4} \mathrm{O}_{8}$ spent fuel transforms to a $\mathrm{U}_{4} \mathrm{O}_{9}$ lattice with non-zero values for $\mathrm{P}_{V}, \mathrm{P}_{\mathrm{S}}$, and $\mathrm{P}_{\mathrm{I}}$ densities.

After the $\mathrm{U}_{4} \mathrm{O}_{9}$ lattice phase is attained, current spent fuel experiments show a $\mathrm{O} / \mathrm{U}$ of $\sim 2.4\left(\mathrm{U}_{4} \mathrm{O}_{9}\right.$ ideally is $\mathrm{O} / \mathrm{U}$ of 2.25), thus there exists an excess $\mathrm{O} / \mathrm{U}$ of $\sim 0.15$, hence, excess oxygen in the $\mathrm{U}_{4} \mathrm{O}_{9}$ lattice. Atomistically, this amount of oxygen is more than sufficient to create a $\mathrm{U}_{3} \mathrm{O}_{7}\left(\mathrm{U}_{3} \mathrm{O}_{7}\right.$ ideally is $\mathrm{O} / \mathrm{U}$ of 2.33) phase; however, a $\mathrm{U}_{3} \mathrm{O}_{7}$ lattice phase has not been observed in the samples from existing low temperature spent fuel oxidation experiments. Thus, the spent fuel $\mathrm{U}_{4} \mathrm{O}_{9}$ phase is a metastable energy phase relative to $\mathrm{U}_{3} \mathrm{O}_{7}$. Equation (3-14) could be readily modified to represent the entropy rate for a $\mathrm{U}_{4} \mathrm{O}_{9}$ to $\mathrm{U}_{3} \mathrm{O}_{7}$ phase transformation by replacing the previous " 48 " by " 49 ", the previous " 49 " by " 37 " and minor changes in numerical coefficients (analogs io expression (3.13)). This development will not be treated here.

The next expected spent fuel phase is $\mathrm{U}_{3} \mathrm{O}_{8}$, which will require additional oxygen to be transported into the $\mathrm{U}_{4} \mathrm{O}_{9}$ lattice for the transformation to occur. At the present time, there is uncertainty about the path to $\mathrm{U}_{3} \mathrm{O}_{8}$; i.e., does $\mathrm{U}_{3} \mathrm{O}_{8}$ form with the addition of sufficient oxygen directly from the nonstoichiometric $\mathrm{U}_{4} \mathrm{O}_{9}$ lattice, or is there an intermediate temporary phase; perhaps $\mathrm{U}_{3} \mathrm{O}_{7}$ and then to $\mathrm{U}_{3} \mathrm{O}_{8}$. Because $\mathrm{U}_{3} \mathrm{O}_{7}$ is a stoichiometric accessible phase from the $\mathrm{U}_{4} \mathrm{O}_{9}$ lattice with a O/U of $\sim 2.4$, its potential intermediate existence will be neglected and equation (3-14) rewritten to represent the thermodynamic energy rates for a transformation directly from $\mathrm{U}_{4} \mathrm{O}_{9}$ lattice to the $\mathrm{U}_{3} \mathrm{O}_{8}$ lattice. This is done by changing in equation (3-14) the existing " 48 " to " 49 " and the previous " 49 " to " 38 "; and the numerical coefficients to satisfy lattice-mass balance relationships are given by the following functional expressions:

$$
\begin{aligned}
& \left.\left.\left.\left.\dot{\bar{P}}_{38}\right] \Leftrightarrow-\dot{\bar{P}}_{49}\right] ; \dot{\bar{P}}_{\mathrm{V}}\right]_{38} \Leftrightarrow-\dot{\bar{P}}_{\mathrm{V}}\right]_{49} \\
& \left.\left.\dot{\bar{P}}_{38}\right] \Leftrightarrow-\dot{\bar{P}}_{\mathrm{O}}\right]_{49}-\dot{\bar{P}}_{\mathrm{O}_{38}} \\
& \left.\left.\left.\left.\dot{\bar{P}}_{\mathrm{I}}\right]_{38} \Leftrightarrow-\dot{\bar{P}}_{\mathrm{I}}\right]_{49} ; \dot{\bar{P}}_{\mathrm{S}}\right]_{38} \Leftrightarrow-\dot{\bar{P}}_{\mathrm{S}}\right]_{49}
\end{aligned}
$$

Then the thermodynamics for the entropy energy rate of $\mathrm{U}_{4} \mathrm{O}_{9}$ to $\mathrm{U}_{3} \mathrm{O}_{8}$ transformations is:

$$
\begin{aligned}
\left.\int_{R} \Delta_{\eta} \varepsilon \dot{\eta} *\right]_{49}^{38} d \underline{x}=\int_{R}\left(\left(\sigma_{i j} L_{i j}\right]_{49}^{38}-\sigma_{i j} L_{i j o}\right]_{49}^{38} \\
\left.\left.+\Delta_{P_{49}} \varepsilon+\Delta_{P_{0}} \varepsilon-\Delta_{P_{38}} \varepsilon\right) \dot{\bar{P}}_{38}\right] \\
\left.\left.\left.+\left(\sigma_{i j} L_{i j o}\right]_{49}^{38}-\Delta_{P_{0}} \varepsilon\right]_{49}^{38}\right) \dot{\bar{P}}_{\mathrm{O}}\right]_{38} \\
\left.\left.\left.+\left(\sigma_{i j} L_{i j l}\right]_{49}^{38}-\Delta_{P_{I}} \varepsilon\right]_{49}^{38}\right) \dot{\bar{P}}_{\mathrm{I}}\right]_{38} \\
\left.\left.\left.+\left(\sigma_{i j} L_{i j j_{S}}\right]_{49}^{38}-\Delta_{P_{S}} \varepsilon\right]_{49}^{38}\right) \dot{\bar{P}}_{\mathrm{S}}\right]_{38} \\
\left.\left.\left.+\left(\sigma_{i j} L_{i j}\right]_{49}^{38}-\Delta_{P_{V}} \varepsilon\right]_{49}^{38}\right) \dot{\bar{P}}_{\mathrm{V}}\right]_{38} \\
\\
\left.\left.-\partial_{i}\left(\Delta_{P_{K}} \varepsilon\right) P_{K}\right] v_{K i}\right) d \underline{x}
\end{aligned}
$$

For homogeneous phase transformations, the full nonequilibrium thermodynamic rate response model from equation (3-16) would couple functionally the nine rate flux functions $\left(\dot{\bar{P}}_{38}\right]$, $\left.\left.\left.\left.\left.\left.\dot{\bar{P}}_{\mathrm{O}}\right]_{38}, \dot{\bar{P}}_{\mathrm{I}}\right]_{38}, \dot{\bar{P}}_{\mathrm{S}}\right]_{38}, \dot{\bar{P}}_{\mathrm{V}}\right]_{38}, \mathrm{P}_{\mathrm{O}}\right] v_{\mathrm{O}_{\mathrm{i}}}, \mathrm{P}_{\mathrm{I}}\right] v_{\mathrm{I}_{\mathrm{i}}}$, $\left.\left.\mathrm{P}_{\mathrm{S}}\right] \mathrm{V}_{\mathrm{S}_{\mathrm{i}}}, \mathrm{P}_{\mathrm{V}}\right] \mathrm{V}_{\mathrm{V}_{\mathrm{i}}}$ ) with their conjugate functional operators (coefficients) in equation (3-16) in a nine-by-nine matrix (counting the four vector fluxes as vector equations, it would be tensorially a 21 by 21 matrix). This type of model was developed in reference [3] for the simplified case of homogeneous phase transformations. The nonequilibrium rate equations for the spatially inhomogeneous phase transformation problem will be developed in the following section. The phase transformation equations are first derived across propagating surfaces which separate different uranium oxide crystallographic lattice structures. 


\section{INHOMOGENEOUS OXIDATION PHASE} TRANSFORMATIONS

\section{Kinematics and Energetics Across a Propagating Phase Front}

In the previous review sections, the spatial dependence of the density functions $P_{\mathrm{MN}}$ and $P_{\mathrm{K}}$ were assumed to be continuous. In this section these density functions are assumed to be spatially discontinuous. The discontinuity for the functions is taken to be across an idealized smooth surface $\partial R]$ in the spatial domain $R_{0}+\partial R_{0}$. For the $\mathrm{U}_{4} \mathrm{O}_{8}$ to $\mathrm{U}_{4} \mathrm{O}_{9}$ phase transformation, the surface is denoted as $\partial R]_{48}^{49}$ and the $\mathrm{U}_{4} \mathrm{O}_{9}$ to $\mathrm{U}_{3} \mathrm{O}_{8}$ phase surface is denoted as $\partial R]_{49}^{38}$. Let surface $\left.\partial R\right]_{48}^{49}$ have a propagation velocity denoted by $\underline{V}_{48}$ and surface $\partial R]_{49}^{38}$ have a propagation velocity denoted by $\underline{\mathrm{V}}_{49}$.

Consider first an arbitrary subdomain $R+\partial R$ of $R_{\mathrm{o}}+\partial R_{\mathrm{o}}$ that contains a propagating discontinuity surface $\partial R]_{48}^{49}$. It follows that in $R+$ $\partial R$, lattice structures of $\mathrm{U}_{4} \mathrm{O}_{8}$ and $\mathrm{U}_{4} \mathrm{O}_{9}$ exist, and are instantaneously separated by surface $\partial R]_{48}^{49}$. In domain $R+\partial R$, consider the additive existence of density functions $\mathrm{P}_{48}$ ] and $\mathrm{P}_{49}$ ]; and their time rates of change. For the case of no discontinuity surface $\partial R]_{48}^{49}$, for any arbitrary time $t$, the result is

$$
\begin{aligned}
& \left.\left.\int_{R} \partial_{t}\left(P_{48}\right]+P_{49}\right]\right) d R \\
& \left.\left.\quad+\int_{\partial R}\left(P_{48}\right]+P_{49}\right]\right) v_{i} n_{i} d(\partial R)=0
\end{aligned}
$$

which follows because each four uranium, atoms occupy a lattice and the lattice is either $\mathrm{U}_{4} \mathrm{O}_{8}$ or $\mathrm{U}_{4} \mathrm{O}_{9}$. Next, consider the case where $R+\partial R$ contains surface $\partial R$ ] with unit normal vector $n$ propagating at velocity $\underline{\mathrm{V}}_{48}$ into a $\mathrm{U}_{4} \mathrm{O}_{8}$ spatial domain, then equation (4-1) still must equal zero, but it will contain additional terms for $\partial R$ ], i.e.

$$
\begin{aligned}
& \left.\left.\int_{R} \partial_{i}\left(P_{48}\right]+P_{49}\right]\right) d R \\
& \left.\left.\quad+\int_{\partial R}\left(P_{48}\right]+P_{49}\right]\right) \mathrm{v}_{i} n_{i} d(\partial R)
\end{aligned}
$$

$$
\begin{aligned}
& \left.\left.\left.+\int_{\partial R]^{+}}\left(P_{48}\right]+P_{49}\right]\right)\left(\mathrm{v}_{i}^{+}-V_{i 48}\right) n_{i}^{+} d(\partial R]\right) \\
& \left.\left.\left.+\int_{\partial R]^{-}}\left(P_{48}\right]+P_{49}\right]\right)\left(\mathrm{v}_{i}^{-}-V_{i 48}\right) n_{i}^{-} d(\partial R]\right)=0
\end{aligned}
$$

where the $(+)$ and $(-)$ superscripts denote opposite sides of the surface $\partial R$ ]. Because the front $\partial R$ ] divides $R+\partial R$ into subdomains of only $\mathrm{U}_{4} \mathrm{O}_{8}$ or $\mathrm{U}_{4} \mathrm{O}_{9}$, then equation (4-1) can be used to reduce equation (4-2) to a surface integral condition (jump condition) only on $\partial R$ ] that is equal to zero; and can be written as

$$
\left.\left.\left.\left.\int_{\partial R]}\left(P_{48}\right]+P_{49}\right]\right)\left(\mathrm{v}_{i}-V_{i 48}\right)\right]_{-}^{+} n_{i} d(\partial R]\right)=0
$$

where $n_{i}$ is directed parallel to $n_{i}^{+}$and the bold bracket (]$)_{-}^{+}$is a shorthand notation for combining the difference of the integrand on opposites of the front $\partial R]$. Finally, since the domain $R+\partial R$ was arbitrary in extend, it follows that the integrand of equation (4-3) is zero at all points along $\partial R$ ]; hence, the pointwise difference condition along $\partial R]$ in $R_{0}+\partial R_{0}$ is

$$
\begin{aligned}
\left(P_{48}\right] & \left.\left.\left.+P_{49}\right]\right)\left(\mathrm{v}_{i}-V_{i 48}\right)\right]_{-}^{+} n_{i} \\
& =0 \text { for } \mathrm{x} \text { on } \partial R]
\end{aligned}
$$

which appears as a standard shock front discontinuity condition across a propagating surface. For the conditions defined above, the surface $\partial R]$ moves into the $\mathrm{U}_{4} \mathrm{O}_{8}$ spatial domain at velocity $V_{i 48}$ and $n_{i}$ points outward (positive) from the $\mathrm{U}_{4} \mathrm{O}_{9}$ domain; thus, equation (4-4a) can be written as

$$
\left.\left.\left(P_{48}^{+}\right]\left(\mathrm{v}_{i}^{+}-V_{i 48}\right)-P_{49}^{-}\right]\left(\mathrm{v}_{i}^{-}-V_{i 48}\right)\right) n_{i}=0(4-4 \mathrm{~b})
$$

because $\mathrm{P}_{49}^{+}$] is zero on the $\mathrm{U}_{4} \mathrm{O}_{8}$ side of surface $\partial R$ ] and $\mathrm{P}_{48}^{-}$] is zero on the $\mathrm{U}_{4} \mathrm{O}_{9}$ side of surface $\partial R]$. For the case of $\mathrm{v}_{i}^{+}$equal to zero (the $\mathrm{U}_{4} \mathrm{O}_{8}$ lattice fixed), equation (4-4b) can be expressed as the number of $P_{49}^{-}$] lattices in terms of the number of $P_{49}^{+}$] per unit spatial volume across $\left.\partial R\right]$ as

$$
\left.\left.\left.P_{49}^{-}\right]=-P_{48}^{+}\right]\left(V_{j 48} n_{j} /\left(-\mathrm{v}_{i}\right\}_{-}^{+}-V_{i 48}\right) n_{i}\right)
$$


where $\left.\mathrm{v}_{i}\right\}_{-}^{+}$is the discontinuity in lattice velocity across the propagating phase front $\partial R]$. Physically, the velocity fraction term in equation $(4-4 c)$ is the volume change across the phase front to correctly evaluate the number of lattice cells per unit spatial volume. In the case of a $\mathrm{U}_{4} \mathrm{O}_{8}$ transformation to $\mathrm{U}_{4} \mathrm{O}_{9}$, the stress-free lattice dimensions decrease, and the relative velocity $\left.v_{i}\right\}_{-}^{+}$ $n_{i}$ is negative across $\partial R$ ] for the normal vector $n_{i}$ directed from the $\mathrm{U}_{4} \mathrm{O}_{9}$ side into the $\mathrm{U}_{4} \mathrm{O}_{8}$ side. Thus, there are more $P_{49}$ ] lattice cells per unit spatial volume than there were $P_{48}$ ] lattice cells per unit spatial volume created as the oxidation front propagates into the $\mathrm{U}_{4} \mathrm{O}_{8}$ spatial domain.

An analysis similar to that completed for lattice densities across a front $\partial R$ ] can also be done for the atom/vacancy densities $P_{\mathrm{K}}$ ]. However, each density function of the set $P_{\mathrm{K}}$ ] can have a singular gain/loss representation across a oxidation front; in particular, consider the atomic oxygen density $P_{0}$ ] which must change as $\mathrm{U}_{4} \mathrm{O}_{8}$ takes a lattice oxygen atom to create $\mathrm{U}_{4} \mathrm{O}_{9}$. Thus, the analog of equation (4-3) for the $\left.P_{\mathrm{K}}\right]$

$$
\begin{gathered}
\left.\left.\left.\int_{\partial R]} F_{K}\right]\left(v_{i}+v_{K_{i}}-V_{i 48}\right)\right]_{-}^{+} n_{i} d(\partial R]\right) \\
\left.\left.\left.=-\int_{\partial R]} \dot{p}_{K}\right] n_{i}\right]_{-}^{+} n_{i} d(\partial R]\right)
\end{gathered}
$$

where

$$
\left.\left.\left.\left.\dot{p}_{K}\right] n_{i}\right]_{-}^{+} n_{i}=\dot{p}_{K}^{+}\right] n_{i}^{+} n_{i}-\dot{p}_{K}^{-}\right] n_{i}^{-} n_{i}
$$

and since $n_{i}^{+} n_{i}=-1$ and $n_{i}^{-} n_{i}=+1$ for the unit normal vector for $n_{i}$ directed from the $R^{-}\left(\mathrm{U}_{4} \mathrm{O}_{9}\right)$ subdomain. The two-sided gain/lost rate terms provide a means of addressing the lattice source of the species, $\left.\dot{p}_{K}^{+}\right]$from $\mathrm{R}^{+}$and $\left.\dot{p}_{K}^{-}\right]$from $R^{-}$sides of $\partial R]$. For example, the oxygen atoms for $\mathrm{U}_{4} \mathrm{O}_{8}$ to $\mathrm{U}_{4} \mathrm{O}_{9}$ conversion can be from the excess lattice oxygen atoms in the $\mathrm{U}_{4} \mathrm{O}_{9}$ lattice $\left(R^{-}\right.$side of $\left.\left.\partial R\right]\right)$ or from those in the $\mathrm{U}_{4} \mathrm{O}_{8}$ lattice $\left(R^{+}\right.$side of $\left.\left.\partial R\right]\right)$ or a combination thereof. The lattice source of oxygen atoms is partly determined by the solidstate solubility limit of oxygen atoms in the lattice structure, and this will affect the energetics of the oxidation process. Finally, regardless of the source, for each $\mathrm{U}_{4} \mathrm{O}_{9}$ lattice created from a $\mathrm{U}_{4} \mathrm{O}_{8}$ lattice, one oxygen atom is required for stoichiometric $\mathrm{U}_{4} \mathrm{O}_{9}$; and additional oxygen atoms are required for hyper-stoichiometric $\mathrm{U}_{4} \mathrm{O}_{9}$ lattice with an $\mathrm{O}$ to $\mathrm{U}$ ratio of $\sim 2.4$. Thus, there is a net positive flux of oxygen atoms to $\partial R$ ], which must equal $\left.\left.\dot{p}_{o}^{+}\right]+\dot{p}_{o}^{-}\right]$on $\left.\partial R\right]$. For the case of nonstoichiometric $\mathrm{U}_{4} \mathrm{O}_{9}$ with an oxygen to uranium ratio of $\sim 2.4$, the sum of $\left.\dot{p}_{o}^{+}\right]$and $\left.\dot{p}_{o}^{-}\right]$must be about 1.6 oxygen atoms per unit crystal lattice site of $\mathrm{U}_{4} \mathrm{O}_{8}$ transformed into a lattice site of $\mathrm{U}_{4} \mathrm{O}_{9}$. For a species density " $K$ " which has zero net flux to surface $\partial R$ ], then the terms $\left.\dot{p}_{K}^{+}\right]$and $\left.\dot{p}_{K}^{-}\right]$are each assumed to be zero.

From equation (4-5a), and because surface segment $\partial R$ ] is part of an arbitrary subdomain $R+$ $\partial R$ in $R_{0}+\partial R_{0}$, the pointwise form across surface $\partial R]$ is

$$
\begin{aligned}
& \left.\left(P_{K}^{+}\right]\left(\mathrm{v}_{i}^{+}+v_{K_{i}}^{+}-V_{i 48}\right)+\dot{p}_{K}^{+}\right] n_{i}^{+} \\
& \left.\left.=P_{K}^{-}\right]\left(\mathrm{v}_{i}^{-}+v_{K_{i}}^{-}-V_{i 48}\right)-\dot{p}_{K}^{-}\right] n_{i}^{-}
\end{aligned}
$$

For the case of stationary $\mathrm{U}_{4} \mathrm{O}_{8}\left(\mathrm{v}_{i}^{+}=0\right)$, the equation (4-6a) can be solved for the flux of species " $\mathrm{K}$ " from the $\mathrm{U}_{4} \mathrm{O}_{9}$, or $\mathrm{R}^{-}$side, to $\partial R$ ], which is

$$
\begin{aligned}
& \left.\left.P_{K}^{-}\right]\left(\mathrm{v}_{i}\right\}_{+}^{-}+v_{K_{i}}^{-}-v_{i 48}\right) n_{i} \\
& \left.\left.\left.=\left(P_{K}^{+}\right]\left(v_{K_{i}}^{+}-V_{i 48}\right)+\dot{p}_{K}\right] n_{i}\right]\right) n_{i}
\end{aligned}
$$

or this can also be written as

$$
\begin{aligned}
& \left.\left.\left.P_{K}^{-}\right]\left(\mathrm{v}_{i}\right\}_{-}^{+}+V_{i 48}\right) n_{i}=P_{K}^{+}\right] V_{i 48} n_{i} \\
& \left.\left.\left.+\left(P_{K}\right] v_{K_{i}}+\dot{p}_{K}\right] n_{i}\right)\right]_{-}^{+} n_{i}
\end{aligned}
$$

Equation (4-6c) is the form that is believed most useful in developing a nonequilibrium thermodynamic response.

The relative velocity $\left.v_{i}\right]_{-}^{+}$across $\left.\partial R\right]$ can be evaluated with equation (2-4) and the reference elasticity strain measured introduced in equation (2-6) from the following expression; 


$$
\begin{gathered}
\left.\mathrm{v}_{i}\right]_{-}^{+}=\int_{\underline{X}^{-}}^{\underline{X}^{+}} \frac{\cdot}{L_{i \mathrm{NM}}\left(P_{N M}\left|-P_{N M O}\right|\right)}+ \\
\left.\left.L_{i j \mathrm{NM}} \dot{\bar{P}}_{\mathrm{NM}}\right]+L_{i j \mathrm{~K}} \dot{\bar{P}}_{\mathrm{K}}\right] d x_{j}
\end{gathered}
$$

where $P_{\mathrm{NMO}}$ is $P_{\mathrm{NM}}\left(x, 0, q_{0}\right)$ and $X^{+}$is on the $\mathrm{U}_{4} \mathrm{O}_{8}$ side of $\left.\partial R\right]$ and $X^{-}$is on the $\mathrm{U}_{4} \mathrm{O}_{9}$ side of $\partial R$ ]. Equation (4-7) is for a relative velocity measure, hence, the integration can be performed moving with the phase front velocity $V_{i 48}$ to obtain a lattice discontinuity velocity across $\partial R$ ] of

$$
\begin{aligned}
\left.\mathrm{v}_{i}\right\}_{-}^{+} & \left.=\underline{-}_{-i j N M}\left(P_{N M}\left|-P_{N M O}\right|\right)\left(\mathrm{v}_{j}-V_{j 48}\right)\right]_{-}^{+} \\
& \left.\left.+L_{i j N M} P_{N M}\right]\left(\mathrm{v}_{j}-V_{j 48}\right)\right]_{-}^{+} \\
& \left.\left.+L_{i j K} P_{K}\right]\left(\mathrm{v}_{j}+v_{K j}-V_{j 48}\right)\right]_{-}^{+}
\end{aligned}
$$

For the case of a $\mathrm{U}_{4} \mathrm{O}_{9}$ oxidation phase front propagating into a stationary $\mathrm{U}_{4} \mathrm{O}_{8}$ phase, $\left(v_{j}^{+}=0\right)$, equation (4-8) becomes (plus side is $\mathrm{U}_{4} \mathrm{O}_{8}$ and minus side is $\mathrm{U}_{4} \mathrm{O}_{9}$ )

$$
\begin{aligned}
& \left.\mathrm{v}_{i}\right\}_{-}^{+}=-L_{i j 48}\left(P_{48}^{+}\left|-P_{480}^{+}\right|\right)\left(V_{j 48}\right) \\
& \left.-L_{i j 49}\left(P_{49}^{-} \mid-P_{490}^{-}\right)\left(-\mathrm{v}_{j}\right\}_{-}^{+}-V_{j 48}\right) \\
& \left.\left.\left.-L_{i j 48} P_{48}^{+}\right]\left(V_{j 48}\right)-L_{i j 49} P_{49}^{-}\right]\left(-\mathrm{v}_{j}\right\}_{-}^{+}-V_{j 48}\right) \\
& \left.+L_{i j K} P_{K}^{+}\right]\left(v_{K j}^{+}-V_{j 48}\right) \\
& \left.\left.-L_{i j K} P_{K}^{-}\right]\left(-\mathrm{v}_{j}\right\}_{-}^{+}+v_{K j}^{-}-V_{j 48}\right)
\end{aligned}
$$

where the velocity $v_{j}^{-}$on the minus side $\left(\mathrm{U}_{4} \mathrm{O}_{9}\right)$ of the front $\partial R$ ] has been replaced with $\left.-v_{i}\right\}_{-}^{+}$for a stationary $\mathrm{U}_{4} \mathrm{O}_{8}$ phase $\left(\mathrm{v}_{j}^{+}=0\right)$ on the plus side of the front $\partial R$ ]. Note that $\left.v_{i}\right\}_{-}^{+}$is not explicitly evaluated in equation (4-9) since it exists on both sides of the equal sign. Furthermore, the total relative velocity does not decompose in simple "continuum" and "discontinuum" terms in the general tensor form given in equation (4-9). However, results from equations (4-4) through (4-6) can be combined into equation (4-9); which along with the physically reasonable assumption that the crystallographical lattice vectors are narrowly distributed about their corresponding lattice dimensional sizes for $\mathrm{UO}_{2}\left(\mathrm{U}_{4} \mathrm{O}_{9}\right)$ and $\mathrm{U}_{4} \mathrm{O}_{9}$ (i.e., functions $P_{N M} l$ and $P_{N M}$ ] are independent, and the $P_{N M}$ and $P_{K}$ density functions are mathematically represented by Dirac delta functions about their corresponding lattice dimensional vectors on vector domains $\{\underline{a}, \underline{b}, \underline{c}\})$ can be used to reduce equation (4-9) to the form

$$
\begin{aligned}
\left.\mathrm{v}_{i}\right\}_{-}^{+} & \left.\left.=-\mathrm{v}_{i}\right\}_{48}^{49}=L_{i j N M}\right]_{49}^{48} P_{48}^{+} I_{0}^{t} V_{j 48} \\
& \left.\left.+L_{i j N M}\right]_{49}^{48} P_{48}^{+}\right] V_{j 48} \\
& \left.\left.\left.\left.+L_{i j K}\right]_{49}^{48}\left(P_{\mathrm{K}}^{+}\right]\left(v_{K^{j}}^{+} V_{j 48}\right)+\dot{p}_{K}\right] n_{j}\right]_{49}^{48}\right)
\end{aligned}
$$

where

$$
\begin{aligned}
& \left.L_{i j \mathrm{NM}}\right]_{49}^{48} \equiv L_{i j 49}\left(q_{49}\right)-L_{i j 48}\left(q_{48}\right) \\
& \left.P_{N M}^{+}\right|_{0} ^{\mu} \equiv P_{N M}\left(\underline{x}^{+}, t, q_{N M}\right) \mid \\
& -P_{\mathrm{NM}}\left(x^{+}, 0, q_{\mathrm{NM}}\right) \mid \\
& \left.L_{i j K}\right]_{49}^{48} \equiv L_{i j K}\left(q_{49}\right)-L_{i j K}\left(q_{48}\right)
\end{aligned}
$$

and the argument $\left(q_{\mathrm{NM}}\right)$ denotes that the operators $L_{i j N M}$ and $L_{i j K}$, and the density function (Dirac delta functions) are evaluated at the " $N M$ " lattice vector values of $(a, b, c)$. Physically, equation (4-10) is an explicit representation for the velocity discontinuity across propagating oxidation front. This relative velocity has three contributions; the first is the continuum term and results from the elastic strain changes across $\partial R$ ] which are small compared to lattice vector changes for a $\mathrm{U}_{4} \mathrm{O}_{8}$ phase change to $\mathrm{U}_{4} \mathrm{O}_{9}$; the second is a discontinuum term and results from lattice phase dimensional changes (which are significant and are the order of a few percent for $\mathrm{U}_{4} \mathrm{O}_{8}$ to a $\mathrm{U}_{4} \mathrm{O}_{9}$ phase change); and the third is also a discontinuum terms and results from the transport of atomic interstitials, atomic substitutionals, vacancies, and oxygen atoms to the phase change front ( relative size is not know but may be significant because of the hyper-stoichiometry of oxygen $\left(\mathrm{UO}_{2.4}\right.$ in a $\mathrm{U}_{4} \mathrm{O}_{9}$ lattice)). To illustrate only the phase change 
contribution to the relative velocity, assume that the elastic and $P_{K}$ ] contributions to the velocity are negligible. A reference coordinate system $\left(x_{1}, x_{2}, x_{3}\right)$ can be selected such that vector $\underline{a}$ is parallel to $x_{1}, b$ is parallel to $x_{2}$, and $c$ is parallel to $x_{3}$. Also, the direction of $x_{2}$ is assumed locally perpendicular to the surface $\partial R$ ] which is propagating with velocity $V_{i 48}=\left(0, V_{2}, 0\right)$. Then, equations (4-10) and (4-11) give

$$
\begin{aligned}
\left.\mathrm{v}_{i}\right\}_{49}^{48} & =\left(\left(b_{i} e_{213} a_{1} c_{3}\right)_{49}\right. \\
& \left.\left.-\left(b_{i} e_{213} a_{1} c_{3}\right)_{48}\right) P_{48}^{+}\right] V_{2}
\end{aligned}
$$

The $b_{i}(49)$ of the $\mathrm{U}_{4} \mathrm{O}_{9}$ lattice in direction $x_{2}$ is smaller than $b_{i}(48)$ of the $\mathrm{U}_{4} \mathrm{O}_{9}$ lattice, and because the alternating tensor $e_{213}$ component is $(-1)$, the relative velocity across the front is the same sign as the propagation velocity $V_{48}$ of $\partial R$ ]. For $U_{4} \mathrm{O}_{8}$ to a $\mathrm{U}_{4} \mathrm{O}_{9}$ phase transformation, this positive relative velocity is consistent with a volume decrease of forming $\mathrm{U}_{4} \mathrm{O}_{9}$ lattices from $\mathrm{U}_{4} \mathrm{O}_{8}$ lattices. From equation (4-12), information is not provicied about the transverse components of a potential velocity discontinuity across $\partial R$ ]. This could be evaluated based on the two other component forms of the $L_{i j N M}$ operators. A discussion of which becomes complex since microcrack size fractures have been observed to form in the $\mathrm{U}_{4} \mathrm{O}_{9}$ phase. However, a microcrack deformation mechanism has not been included in this kinematic analysis.

The above developments addressed the phase change of $\mathrm{U}_{4} \mathrm{O}_{8}$ to $\mathrm{U}_{4} \mathrm{O}_{9}$. Similar equations can be written for $\mathrm{U}_{4} \mathrm{O}_{9}$ oxidation to $\mathrm{U}_{3} \mathrm{O}_{8}$. These equations are the same as above with " 49 " replaced by a " 38 " lattice structure identifier, and then " 48 " replaced by a " 49 " lattice structure identifier. In the case of $\mathrm{U}_{3} \mathrm{O}_{8}$, there is a significant volume increase $(\sim 30 \%)$ in forms from $\mathrm{U}_{4} \mathrm{O}_{9}$; thus, the velocity discontinuity $\underline{v}\}_{38}^{49}$ normal to the front is negative as the $\mathrm{U}_{3} \mathrm{O}_{8}$ lattice increases in volume behind a phase front. Because of the phase change volume increase, it is expected that a large number of microcracks would occur and that the $\mathrm{U}_{3} \mathrm{O}_{8}$ spent fuel would potentially be a high porosity waste form with low strength that may flake to micron sized particulates (powders). This is consistent with the above kinematic equations; however, as noted above, the kinematic equations do not include a microcrack dependent deformation response, and therefore are not complete. Moreover, for the particular case of spent fuel degradation, a microcrack response model is not of special importance because experimental observations can be performed to characterize the deformation state of the $\mathrm{U}_{3} \mathrm{O}_{8}$ formed from isolated and composite (fragments) grains of $\mathrm{U}_{4} \mathrm{O}_{9}$.

The next topic in this oxidation front analysis is potential stress discontinuity conditions. For shock fronts the stress discontinuity is derived from the momentum equation and is equal to the discontinuity in the momentum density across the propagating shock front. For a propagating oxidation phase front, a similar derivation can be performed; however, the discontinuity in the momentum density is assumed to be small and neglectable. By neglecting the momentum density, and since the effects of a possible interfacial surface energy between oxides of uranium has not been included, the stress equilibrium equation implies that the stress traction across the oxidation front must be continuous; thus,

$$
\begin{aligned}
& \left.\sigma_{i j} n_{j}\right]_{49}^{48}=0 \\
& \text { across a } \partial R \text { ] oxidation front }
\end{aligned}
$$

Note, that this does not mean that the stress is zero nor that all individual components of the stress tension are continuous across a propagating oxidation front.

The final part of an oxidation front analysis in this section is for the energetic components to the formation of $\mathrm{U}_{4} \mathrm{O}_{9}$ from $\mathrm{U}_{4} \mathrm{O}_{8}$ and $\mathrm{U}_{3} \mathrm{O}_{8}$ from $\mathrm{U}_{4} \mathrm{O}_{9}$. The starting point is equation (3-4), and the integral representations given in equations (3-5) through (3-7). The arbitrary spatial domain $R+\partial R$ now contains a surface $\partial R$ ], across which functional discontinuities in the energy can arise. Thus, the domain $R$ is subdivided into volume $R^{+}$ and $R$ - subsets by $\partial R$ ]; and in each volume subset equation (3-8) will apply. Across the propagating surface $\partial R$ ] with velocity $Y$, which divides the volume subsets, the following equation applies

$$
\begin{gathered}
\left.\left.\int_{\partial R]}\left(\varepsilon(f)\left(v_{i}-V_{i}\right)+\Delta_{P K} \varepsilon(f) P_{K}\right] v_{K i}\right)\right]_{-}^{+} n_{i} d \underline{x} \\
\left.\left.=\int_{\partial R]} \sigma_{i j} v_{j}\right]_{-}^{+} n_{i}+h_{i}\right]_{-}^{+} n_{i} d x
\end{gathered}
$$

For the case of $\mathrm{U}_{4} \mathrm{O}_{8}$ in volume $R^{+}$and $\mathrm{U}_{4} \mathrm{O}_{9}$ in volume $R^{-}, V_{i}$ becomes $V_{i 48}$, and the lattice density function $P_{48}$ is non-zero in $R^{+}$and $P_{49}$ is non-zero in $R^{-}$. 
In order to have a functional dependence on each of the function arguments of $\varepsilon(f)$ as given in equation (3-1), the functional expansion of equation (3-3) is extended to include contributions from the entropy function $\eta$ and the continuum strain ter.sor $\gamma_{i j}$;

$$
\begin{aligned}
\varepsilon\left(f^{\prime}\right) & \equiv \varepsilon_{0}+\Delta_{\eta} \varepsilon\left(\eta-\eta_{0}\right) \\
& \left.+\Delta_{\gamma_{i j}} \varepsilon\left(\gamma_{i j}\left|-\gamma_{i j}\right|_{0}\right)+\Delta_{P_{N M}} \varepsilon P_{N M}\right] \\
& \left.+\Delta_{P_{K}} \varepsilon P_{K}\right]
\end{aligned}
$$

where the lattice reference energy state is evaluated in terms of an arbitrary internal energy $\varepsilon_{0}$, arbitrary entropy $\eta_{0}$, and strain state $\left.\gamma_{i j}\right|_{0}$. Substituting equation (4-15) into (4-14) and rearranging terms, the following pointwise condition across $\partial R$ ] can be obtained.

$$
\begin{aligned}
& \left.\left(\varepsilon_{o}+\Delta_{\eta} \varepsilon\left(\eta-\eta_{o}\right)\right)\left(v_{i}-v_{i 48}\right)\right]_{49}^{48} n_{i} \\
& \left.\left.=\left(\sigma_{i j} v_{i}\right)\right]_{49}^{48} n_{j}+h_{k}\right]_{49}^{48} n_{k} \\
& \left.-\left(\Delta \gamma_{i j} \varepsilon\left(\gamma_{i j} \mid-\gamma_{i j} l_{o}\right)\left(v_{k}-v_{k 48}\right)\right)\right]_{49}^{48} n_{k} \\
& \left.\left.-\left(\Delta_{P_{N M}} \varepsilon P_{N M}\right]\left(v_{k}-v_{k 48}\right)\right)\right]_{49}^{48} n_{k} \\
& \left.\left.-\left(\Delta_{P_{K}} \varepsilon P_{K}\right]\left(v_{k}+v_{K k}-v_{k 48}\right)\right)\right]_{49}^{48} n_{k}
\end{aligned}
$$

where terms such as $\varepsilon_{0}^{+}, \varepsilon_{0}^{-}, \eta_{0}^{+}, \eta_{0}^{-},\left.\gamma_{i j}\right|_{0} ^{+}$, and $\left.\gamma_{i j}\right|_{0} ^{-}$ are arbitrary reference state functions for the two lattice structures of $P_{48}$ and $P_{49}$ adjacent to surface $\partial R$ ]. In order to simplify and eliminate several terms from equation (4-16) the previous assumption is applied that the velocity $\mathrm{v}_{i}^{+}$on the $R^{+}$side of $\partial R$ ] is zero, and the reference state functions are evaluated such that

$$
\begin{aligned}
& \varepsilon_{0}^{+}=0 \\
& \eta_{0}^{+}=\eta^{+} \\
& \left.\gamma_{i j}^{+}\right|_{0}=\gamma_{i j}^{+} \\
& \varepsilon_{0}^{-}=\Delta_{\eta} \varepsilon^{-}\left(\eta_{0^{-}}-\eta^{+}\right) \\
& \gamma_{i j}^{-} l_{0}=0
\end{aligned}
$$

Then, equation (4-16) can be written as

$$
\begin{aligned}
& \left.\left(\Delta_{\eta} \varepsilon^{-}\left(\eta^{-}-\eta^{+}\right)\left(v_{i}\right\}_{-}^{+}+V_{i 48}\right) n_{i}-h_{i}\right]_{49}^{48} n_{i} \\
& \left.=\sigma_{i j}-\left(\mathrm{v}_{i}{ }_{-}^{+}+\mathrm{v}_{i}\right]_{-}^{+}\right) n_{i} \\
& \left.-\Delta_{\gamma i j} \varepsilon^{-} \gamma_{i j}-1\left(\mathrm{v}_{k}\right\}_{-}^{+}+\mathrm{V}_{k 48}\right) n_{k} \\
& +\left(\Delta_{P_{48}} \varepsilon^{+} P_{48}^{+}\right] \mathrm{V}_{i 48} \\
& \left.\left.-\Delta_{P_{49}} \varepsilon^{\left.-P_{49}^{-}\right]}\left(-\mathrm{v}_{i}\right\}_{-}^{+}-V_{i 48}\right)\right) n_{i} \\
& -\left(\Delta_{P_{K}} \varepsilon^{+} P_{K}^{+}\right]\left(v_{K i}^{+}-V_{i 48}\right) \\
& \left.\left.\left.-\Delta_{P_{K}} \varepsilon-P_{K}\right]\left(-v_{i}\right\}_{-}^{+}+v_{K} \bar{i}_{i}-V_{i 48}\right)\right) n_{i}
\end{aligned}
$$

The continuum stress work term can be eliminated by assuming that the oxidation continuum strain rate processes occur at thermodynamic equilibrium (see equation 3-11), which implies that

$$
\sigma_{i j}^{-}=\Delta_{\gamma i j} \varepsilon^{-}
$$

In addition to equation (4-19), an expression developed for the continuum strain tensor across a propagating shock front ${ }^{[5]}$ can be used (which is also true across a propagating oxidation front), namely;

$\left.\gamma_{i j}=1 / 2\left(v_{i} I_{-}^{+} n_{j}+v_{j} I_{-}^{+} n_{i}\right) /\left(v_{k}\right\}_{-}^{+}+V_{k 48}\right) n_{k}$

to show that

$\left.\sigma_{i j}^{-}\left(\left.v_{i}\right|_{-} ^{+}\right) n_{j}=\Delta_{\gamma i j} \varepsilon^{-} \gamma_{i j} \mid\left(v_{k}\right]_{-}^{+}+V_{k 48}\right) n_{k}$

Equation (4-18) can be further simplified by substituting results from equation (4-4) to replace expressions containing $P_{49}$ ], and from (4-10) to replace the discontinuum relative velocity $\left.v_{i}\right]_{-}^{+}$term in the remaining part of the stress work expression. With these substitutions, equation (4-18) can be written as

$$
\begin{gathered}
\left.\left(\Delta_{\eta} \varepsilon^{-}\left(\eta^{-}-\eta^{+}\right)\left(\mathrm{v}_{i}\right\}_{-}^{+}+V_{i 48}\right) n_{i}-h_{i}\right]_{49}^{48} n_{i}= \\
\left.-\left(\Delta_{P_{48}} \varepsilon^{+}-\Delta_{P_{49}} \varepsilon^{-}\right) P_{48}^{+}\right] \mathrm{V}_{i 48} n_{i} \\
\left.-\sigma_{i k}^{-}\left(L_{i j N M}\right]_{48}^{49} P_{48}^{+}\right] \mathrm{V}_{j 48} n_{k} \\
\left.-\left(\Delta_{P_{\mathrm{K}}} \varepsilon^{+}-\Delta_{P_{\mathrm{K}}} \varepsilon^{-}\right) P_{K}^{+}\right] \mathrm{V}_{i 48} n_{i}
\end{gathered}
$$




$$
\begin{aligned}
& \left.-\sigma_{i j}^{-}\left(L_{i k K}\right]_{48}^{49} P_{\mathrm{K}}^{+}\right] \mathrm{v}_{k 48} n_{j} \\
& \left.\left.-\left(\Delta_{P_{\mathrm{K}}} \varepsilon P_{K}\right] v_{K i}\right)\right]_{48}^{49} n_{i} \\
& \left.\left.-\sigma_{i k}^{-}\left(L_{i j K} P_{K}\right] v_{K j}\right)\right]_{48}^{49} n_{k} \\
& \left.\left.-\Delta_{P_{\mathrm{K}}} \varepsilon \dot{p}_{K}\right] n_{j}\right]_{48}^{49} n_{j}
\end{aligned}
$$

The phase change energy condition of ecuation (4-16) can be written in forms different from that in equation (4-22). This form of equation (4-22) expresses the net dissipation entropy discontinuity $\Delta_{\eta} \varepsilon-\eta *$ across a propagating phase change front, which is the total entropy energy change minus the net heat flux across the front. For thermodynamic processes, this dissipation entropy change is always non-negative. The terms on the right hand side of equation (4-22) provide the chemical potential energy changes from the oxidation phase transformation at the front, the work performed during the phase transformation at the front, and the net chemical potential energy transported by lattice atoms and vacancies to the front. A separation of energy terms across a front such that the thermodynamic energy of the oxidation reaction for the $\mathrm{UO}_{2}$ transformation to $\mathrm{U}_{4} \mathrm{O}_{9}$ is not as readily discerned from equation (4-22) as it was for the homogeneous oxidation case described in equations (3-13) and (3-14). To write such a separation, the rate of change for the singular surface terms $\dot{p}_{K}$ ] of oxygen, interstitial, substitutional, and vacancies concentrations must be expressed in terms of the lattice transformation rate $\left.P_{48}^{+}\right] \mathrm{V}_{i 48} n_{i}$. In the case of oxygen, one oxygen atom is removed at the front for each lattice cell of $\left.P_{48}\right]$ transformed to a $P_{49}$ ] cell; thus,

$$
\begin{aligned}
& \left.\left.\left.\dot{p}_{\mathrm{O}}\right] n_{i}\right] n_{i}=+P_{48}^{+}\right] \mathrm{V}_{i 48 n_{i}} \\
& \left.\left.\left.\left.\dot{p}_{\mathrm{O}}\right] n_{i}\right] n_{i}=-P_{49}^{+}\right]\left(\mathrm{v}_{i}\right\}_{-}^{+}+\mathrm{V}_{i 48}\right) n_{i}
\end{aligned}
$$

However, equation (4-22) combines the chemical potential energy of oxygen on the plus and minus sides of the front. Thus, to simplify equation (422), a mechanistic assumption for the oxygen source will be made; namely, the oxygen supplied for the oxidation reaction is provided from the excess oxygen on the $\mathrm{U}_{4} \mathrm{O}_{9}$ (minus) sides of the front. In addition to this assumption, it will be assumed that the interstitial, substitional, and vacancy concentrations across the oxidation front are conserved; thus

$$
\left.\left.\left.\dot{p}_{\mathrm{I}}\right] n_{i}=\dot{p}_{\mathrm{S}}\right] n_{i}=\dot{p}_{\mathrm{V}}\right] n_{i}=0
$$

Using the results of equation (2-23), and the above discussion of net dissipation entropy energy, equation (4-22) can be written as

$$
\begin{aligned}
& \left.\Delta_{\eta} \varepsilon^{-\eta^{*}}\left(\mathrm{v}_{i}\right\}_{-}^{+}+V_{i 48}\right) n_{i}= \\
& +\left(\left(\Delta_{P_{48}} \varepsilon^{+}+\Delta_{P_{\mathrm{O}}} \varepsilon^{-}-\Delta_{P_{49}} \varepsilon^{-}\right) \delta_{k j}\right. \\
& \left.\left.\left.+\sigma_{i j}^{-} L_{i k N M}\right]_{48}^{49}-\sigma_{i j}^{-} L_{i k O}\left(q_{49}\right)\right) P_{48}^{+}\right] \mathrm{v}_{k 48} n_{j} \\
& \left.-\left(\left(\Delta_{P_{\mathrm{K}}} \varepsilon^{+}+\Delta_{P_{\mathrm{K}}} \varepsilon^{-}\right) \delta_{k j}+\sigma_{i j}^{-} L_{i k K}\right]_{48}^{49}\right) \\
& \left.\left.\quad \times\left(P_{K}^{+}\right]+P_{K}^{-}\right]\right) \mathrm{v}_{k 48} n_{j} \\
& \left.-\left(\Delta_{P_{\mathrm{K}}} \varepsilon \delta_{k j}-\sigma_{i j}^{-} L_{i k K}\right)\right]_{48}^{49} \\
& \left.\left.\times\left(P_{K}^{+}\right] v_{K k}^{+}+P_{K}^{-}\right] v_{K k}^{-}\right) n_{j}
\end{aligned}
$$

Equation (4-24) provides the thermodynamic rates and nonequilibrium thermodynamic forces terms for oxidation across a propagating phase transformation front. This equation is an analog to equation (3-14) for the spatially homogeneous phase transformation of $\mathrm{UO}_{2}$ to $\mathrm{U}_{4} \mathrm{O}_{9}$. The positive and negative signs for the thermodynamic forces are the same because the rate $\left.P_{48}^{+}\right] V_{i 48} n_{i}$ is positive since it is written with normal $n_{i}\left(n_{i}=n_{\bar{i}}=\right.$ $-n_{i}^{+}$and the front velocity $V_{i 48}$ is positive into the $P_{48}^{+}$] spatial domain of a $\mathrm{UO}_{2}$ grain. The following section will use Onsager's approach to relate the thermodynamic rates and conjugate thermodynamic force terms for the case of $\mathrm{U}_{4} \mathrm{O}_{8}$ transforming to $\mathrm{U}_{4} \mathrm{O}_{9}$. The energy change equation for the phase transformation of $\mathrm{U}_{4} \mathrm{O}_{9}$ to $\mathrm{U}_{3} \mathrm{O}_{8}$ would have the same terms as those in equation (4-24); the differences would be the initial " 48 " lattice is replaced by the " 49 " lattice, and the final " 49 " lattice is replaced by the " 38 " lattice.

Equation (4-24) extends the equilibrium thermodynamics developed for treating nonstoichiometric energy contributions. $\left.{ }^{2}, 8\right]$ The equilibrium thermodynamic representations do not address the kinetics (rate) of approaching a thermodynamic equilibrium state. In terms of the above equation, the thermodynamic equilibrium 
state, in terms of Gibbs, ${ }^{[9]}$ would be attained when the coefficients of the ate terms on the right hand side of equation (4-24) are zero. Thus, the oxidation front velocity and diffusion rates would he zero because the thermodynamic forces are all zero at thermodynamic equilibrium.

\section{NONEOUILIBRIUM \\ THERMODYNAMICS PHASE TRANSFORMATIONS}

In the previous section, the dissipation energy rate in equation (4-24) is expressed in terms of a phase transformation front rate tensor $\left.P_{48}^{+}\right] V_{i 48} n_{j}$ and mass transport tensors $\left.P_{K}\right] V_{k 48} n_{j}$ and $\left.\left.P_{K}\right] v_{K k}\right] n_{j}$. The tensor nature for these three rates results from the potential for doing work in the tangential directions of the oxidation surface by deforming the surface and by mass transport on the surface. This requires at an atomic scale the evolution of tangential strains and discontinuities from the formation of lattice defects such as point species, line species, or surface species. These defects are commonly known as vacancies, dislocations, and microcracks. In the following the potential tangential motion from strains and from line and surface lattice defects will not be considered. To analyze discontinuities from the line and surface defect species would require that the relative velocity and deformation expressions introduced in equations (2-1) and (2-5) be extended to include dislocation density and microcrack density terms. The neglect of these terms results in approximations because the shear work is not included and the energetics of lattice defects production are omitted.

The terms will be neglected by using the trace of the tensor $V_{i 48} n_{j}$; which is $V_{i 48} n_{i}$. The other tensor rate for mass transport relative to the lattice will be included in the model for oxidation phase transformation across a front. This will make the thermodynamic model consistent with the relative velocity and deformation representation which included the contributions of lattice size changes from vacancy, interstitial, substitutional, and oxygen concentrations.

With this simplification, the thermodynamic rate terms are $\left.\left.\left.P_{48}^{+}\right] V_{i 48} n_{i},\left(P_{K}^{+}\right]+P_{K}^{-}\right]\right) V_{i 48} n_{i}$, and $\left.\left.\left(P_{K}^{+}\right] v_{K k}^{+}+P_{K}^{-}\right] v_{K k}^{-}\right) n_{j}$. From equation (4-24), the conjugate thermodynamic forces are

$$
\begin{aligned}
\mathbf{T}_{48} & \equiv \Delta_{P_{48}} \varepsilon^{+}+\Delta_{P_{\mathrm{O}}} \varepsilon^{-}-\Delta_{P_{49}} \varepsilon^{-} \\
& \left.+\sigma_{i j}^{-} L_{i j M N}\right]_{48}^{49}-\sigma_{i j}^{-} L_{i j \mathrm{O}}\left(q_{49}\right) \\
\mathbf{T}_{K} & \left.\left.\equiv \sigma_{i j}^{-} L_{i j K}\right]_{48}^{49}-\Delta_{P_{K}} \varepsilon\right]_{48}^{49} \\
\mathbf{T}_{K k j} & \left.\left.\equiv \sigma_{i j} L_{i k K}\right]_{48}^{49}-\Delta_{P_{K}} \varepsilon\right]_{48}^{49} \delta_{k j}
\end{aligned}
$$

where $\mathbf{T}_{48}$ and $\mathbf{T}_{K}$ are scalar thermodynamic forces and $\mathbf{T}_{K k j}$ is a second order tensor thermodynamic force. The Onsager form of first order operator equations to couple the thermodynamic rate terms and their conjugate forces are

$$
\begin{aligned}
\left.P_{48}^{+}\right] V_{i 48} n_{i}=\mathcal{L}_{4848} \mathrm{~T}_{48}+\mathcal{L}_{48 K} \mathrm{~T}_{K} \\
+\mathcal{L}_{48 K k j} \mathrm{~T}_{K k j} \\
\left.\left.\left(P_{K}^{+}\right]+P_{K}^{-}\right]\right) V_{i 48 n_{i}}=\mathcal{L}_{K 48} \mathrm{~T}_{48}+\mathcal{L}_{K K} \mathrm{~T}_{K} \\
\quad+\mathcal{L}_{K K k j} \mathrm{~T}_{K k j} \\
\left.\left.\left(P_{K}^{+}\right] v_{K k}^{+}+P_{K}^{-}\right] v_{K k}\right) n_{j}=\mathcal{L}_{K k j 48} \mathrm{~T}_{48} \\
\quad+\mathcal{L}_{K k j K} \mathrm{~T}_{K}+\mathcal{L}_{K k j K m n} \mathrm{~T}_{K m n}
\end{aligned}
$$

where the set of $\{\mathcal{L}\}$ operators are Onsager coefficients that operate on the thermodynamic forces to provide the thermodynamic rates. These coefficients are phenomenological quantities that can be determined experimentally.

For example, if experiments establish that the rate (kinetics) for the phase transformation propagation velocity of the oxidation front is diffusion controlled, then the thermodynamic force $\mathbf{T}_{K k j}$, which is the diffusion force, is the dominant one and the coefficient $L_{48 K k j}$ can be established. On the other hand, if the phase change energy force $\mathbf{T}_{48}$ is controlling the front velocity of phase changes, then it is $\mathcal{L}_{48} 48$ that experiments will isolate for evaluation.

At the present time, experiments have established that nonstoichiometric oxidation phase changes are occurring in spent fuel. This probably means that fission products from fission events and actinides created from neutron capture/decay processes are stabilizing lattice structures. This suggests that a 
consistent thermodynamic model which represented a dependence on different concentrations of fission products and actinides should be developed for comparisons with experimental data. The concentration of fission products and actinides are represented in an aggregated approximation by the interstitial and substitutional density functions $\left.P_{I}\right]$ and $\left.P_{S}\right]$, respectively. As a first a! pproximation, these two densities should monotonically increase with the number of fission events per unit volume of reactor fuel, which is commonly termed "burnup." Thus, in a linear model, the approximate fission products and actinide concentrations are

$$
\begin{aligned}
& \left.\left.\left.P_{\mathrm{I}}\right]=P_{\mathrm{I}}\right]_{\mathrm{o}}+\Delta_{f} P_{\mathrm{I}}\right] f \\
& \left.\left.\left.P_{\mathrm{S}}\right]=P_{\mathrm{S}}\right]_{\mathrm{o}}+\Delta_{f} P_{\mathrm{S}}\right] f
\end{aligned}
$$

where $f$ is the accumulated fission events (burnup) of the spent fuel, the coefficients of $f$ can be aggregately approximated from radionuclide fission product and actinide data, and the terms with subscript "o" are initial coefficients that can be used to test possible effects of some BWR spent fuels that have several initial percent of burnable poisons such as gadolina.

The difficult part of such a model is that the influence of $\left.P_{I}\right]$ and $P_{S}$ ] on the thermodynamic rates can occur in either the set of Onsager coefficients $\{\mathcal{L}\}$ or the set of thermodynamic forces $\{\mathbf{T}\}$; or both sets. In any of the three possible cases, the general form of equation (5-2) permits one to derive a thermodynamically consistent model to describe burnup effects on the kinetics of oxidation phase transformation in spent fuel. In the following, the approximation given in equation (5-3) is used to represent kinetic influences of burnable poisons in spent fuel and the degree of spent fuel burnup.

For two simple illustrative oxidation response models, consider first the case where the phase front has a constant velocity of propagation into spent fuel grains; and for the second case assume the phase front velocity has a square root of time dependence. In case one, it is assumed that the energy kinetics of force $\mathbf{T}_{48}$ dominates the rate response; and for case two it is assumed that the diffusion kinetics of force $\mathbf{T}_{K k j}$ dominates the rate response. From equations (5-2a) and (5-3), consistent thermodynamic models that have a linear burnup dependence on these thermodynamic forces are given simply by
Case 1

$$
\left.P_{48}^{+}\right] V_{i 48 n_{i}}=\mathcal{L}_{4848}\left(\mathbf{T}_{480}+\Delta_{f} \mathbf{T}_{48} f\right)
$$

Case 2

$$
\left.P_{48}^{+}\right] V_{i 48 n_{i}}=\mathcal{L}_{48 K k j}\left(\mathbf{T}_{K k j o}+\Delta_{f} \mathbf{T}_{K k j} f\right)
$$

In Case 1 , the velocity $V_{i 48}$ has a linear- dependence on burnup fissions $f$. In Case 2 the square root time dependence occurs as a result of the thermodynamic force (the $\Delta \mathrm{P}_{K} \varepsilon$ ] term) decaying in time as the thickness of the oxidation zone around the perimeter of a grain increases in thickness.

These two models were idealized and presented to illustrate the utility of a general formulation that uses nonequilibrium thermodynamics for relationships. It should be clear, however, that this thermodynamic approach must be coordinated with an experimental approach. Only then can the kinetics problem of oxidation and phase transformation of radioactive spent fuel be adequately understood to address performance and degradation issues relative to repository analysis and design.

\section{Acknowledgements}

This work was performed with funds from the Yucca Mountain Site Characterization Project (YMP) and a USDOE/AECL (Canada) International Agreement Program as part of the Civilian Radioactive Waste Management Program by Lawrence Livermore National Laboratory under Contract W-7405-ENG-48. 


\section{References}

1. L.E. Thomas and R.E. Einziger, Mater.

Charact. 28, 149 (1992).

2. R.E. Einziger, L.E. Thomas, H.C.

Buchanan, and R.B. Stout, Proc. of the 3rd

Int. HLRWM Conf. Las Vegas, NV (1992).

3. R.B. Stout, E.J. Kansa, and A.M.

Wijesinghe, Kinematics and

Thermodynamics of Non-Stoichiometric

Oxidation Phase Transitions in Spent Fuel,

Livermore National Laboratory Report

UCRL-JC-110678, January (1993).

4. R.B. Stout, Crystal Lattice Defects 9, 65 (1981).

5. R.B. Stout, in Wave Propagation in

Granular Media, D. Karamonlidis and R.B. Stout eds., p. 51, AMD 101, ASME, New York (1989).

6. L. Onsager, Phys. Rev. 37, 405 (1931); and 38, 2265 (1931).

7. A.L.G. Rees, Chemistry of the Defect Solid State, Methuens Monographs, London (1954).

8. J.S. Anderson in Problems of Nonstoichiometry, A. Rabenau, ed., North Holland and Elsevier (1970) p. 1; Y.P. Jeannin, ibid., p. 77; H. Jagodzinski, ibid., p. 131.

9. J.W. Gibbs, The Scientific Papers of J. Willard Gibbs, Dover, Inc. New York, (1961). 

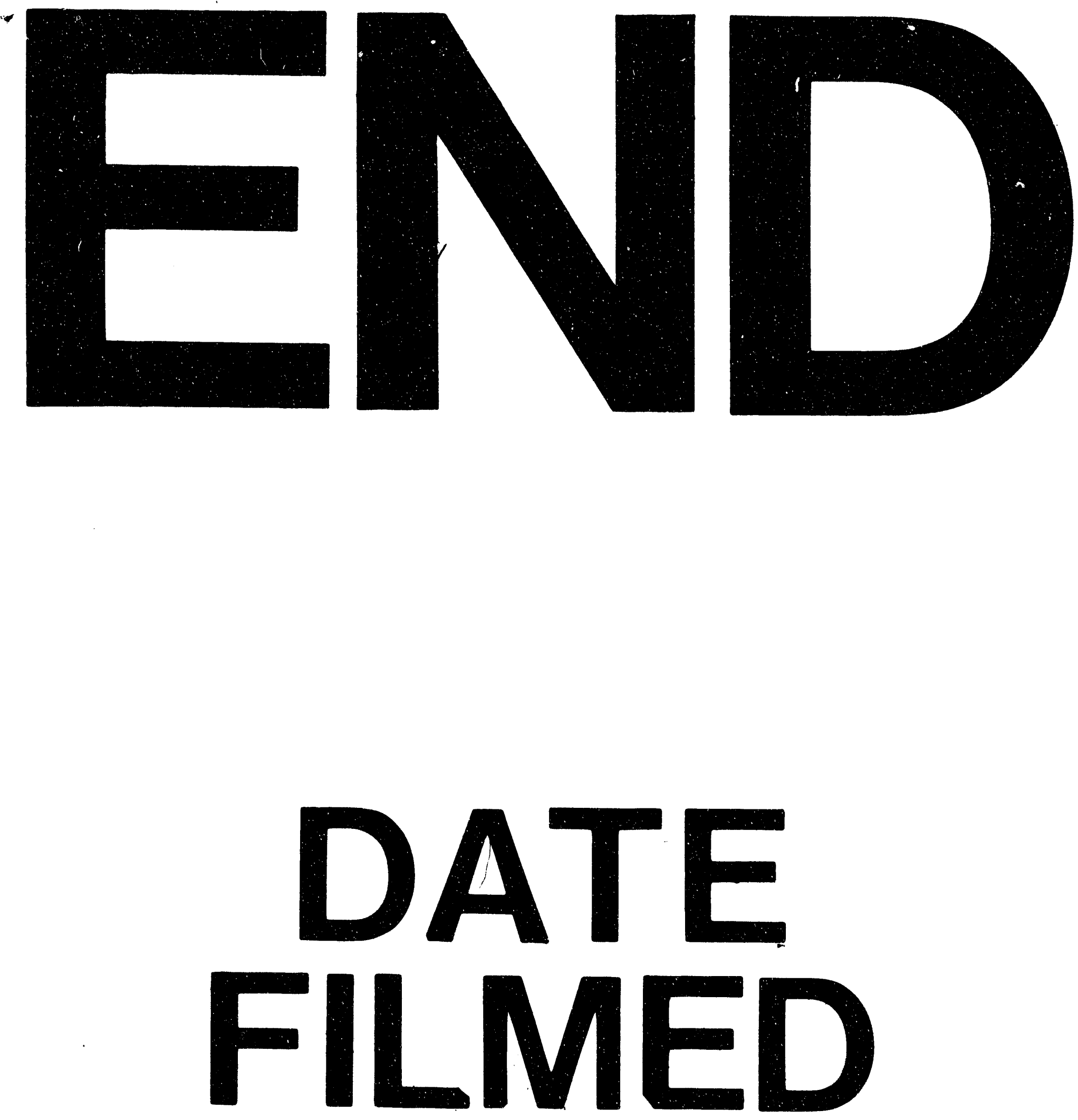

1

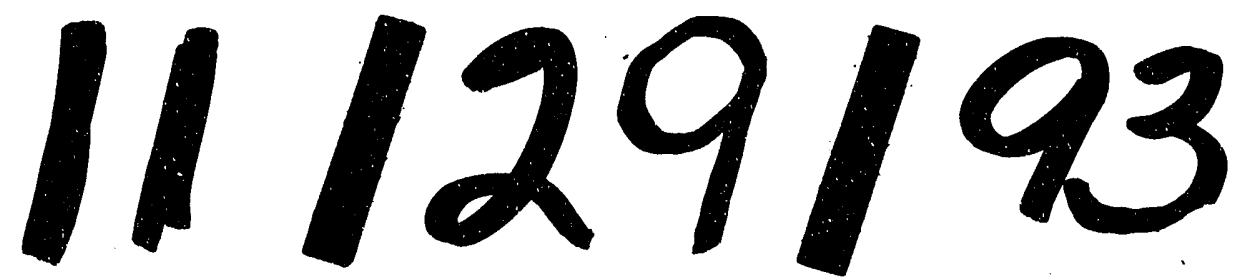


